\title{
An Integrated Consensus Improving Strategy Based on PL-Wasserstein Distance and Its Application in the Evaluation of Network Public Opinion Emergencies
}

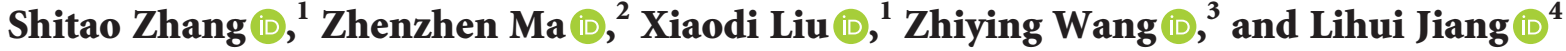 \\ ${ }^{1}$ School of Mathematics \& Physics Science and Engineering, Anhui University of Technology, Ma'anshan 243032, China \\ ${ }^{2}$ School of Economics and Management, Beihang University, Beijing 100191, China \\ ${ }^{3}$ School of Management Science and Engineering, Anhui University of Technology, Ma'anshan 243032, China \\ ${ }^{4}$ College of Artificial Intelligence and Big Data, Hefei University, Hefei 230601, China \\ Correspondence should be addressed to Zhenzhen Ma; zhenzhen5886@163.com
}

Received 22 April 2020; Revised 29 October 2020; Accepted 11 November 2020; Published 1 December 2020

Academic Editor: Peter Giesl

Copyright (C) 2020 Shitao Zhang et al. This is an open access article distributed under the Creative Commons Attribution License, which permits unrestricted use, distribution, and reproduction in any medium, provided the original work is properly cited.

In real life, multiple network public opinion emergencies may break out in a certain place at the same time. So, it is necessary to invite emergency decision experts in multiple fields for timely evaluating the comprehensive crisis of the online public opinion, and then limited emergency resources can be utilized to give priority to respond to the one with the highest crisis. Due to the complexity of network public opinion emergencies and the limited cognition of experts, most of the decision problems for evaluating the network public opinion emergencies are highly uncertain. Also, prior to the selection of the highest crisis, it is preferable that experts reach a high degree of consensus among their assessments or opinions. To address such problems, this paper presents a novel adaptive consensus reaching model for multiattribute group decision making (MAGDM) with probabilistic linguistic decision matrices (PLDMs). First, to quantify the difference between any two probabilistic linguistic term sets (PLTSs) accurately and efficiently, we define a novel distance measure between PLTSs based on the Wasserstein metric. Then, by integrating the defined PLTSs-based Wasserstein (PL-Wasserstein) distance measure into the classical CCSD method, we construct an optimization model for objectively determining attribute weights. Subsequently, we develop the individual cumulative consensus contribution (ICCC) measure and the group consensus measure, respectively, following which is to present an integrated consensus improving strategy that considers both weight-updating (i.e., dynamic weights of experts and attributes) and assessment-adjusting. Finally, the feasibility and the applicability of the proposed approach are illustrated via a real evaluation of network public opinion emergencies. Through comparing with existing probabilistic linguistic MAGDM approaches, the proposed approach offers the advantages in terms of the accurate measurement of information difference and the integrated improvement of consensus efficiency.

\section{Introduction}

According to China Internet Network Information Center (CNNIC) 43th statistical report on Internet development in Beijing (released on the 28th of February, 2019), it follows that as of the end of December, 2018, Chinese netizens reached 829 million, the Internet penetration rate reached 59.6\%, and the usage rate of WeChat friends circle, QQ space, and Weibo as social media reached $83.4 \%, 58.8 \%$, and $42.3 \%$, respectively, among which Sina Weibo monthly active users have reached
462 million (https://www.cnnic.net.cn/hlwfzyj/hlwxzbg/ hlwtjbg/201902/t20190228_70645.htm). Driven by social applications such as WeChat, QQ, and Weibo, after being reported, hyped, and transmitted on the Internet, emergencies in real life are rapidly spread in the society and eventually evolve into hot spots with social influence $[1,2]$. Hot topics that are updated every several minutes have a profound impact on online public opinion hot spots. The huge number of Chinese netizens has also accelerated the fermentation and spread of hot spots, resulting in potentially greater harm [3]. 
For example, a medical-related incident "Wei Zexi Incident" that triggered netizens' attention on the Internet became a focus event of public opinion from April to May 2016. The military dog public opinion monitoring system showed that from April 9 to May 3, 2016, there was 1398077 related public opinion information, of which Weibo accounted for $80.2 \%$ which is the most, followed by news information, $15.9 \%$; forum information was the least, $3.9 \%$. Considering the huge influence and potential hazard of similar network public opinion emergencies, the Chinese governments at all levels have taken the governance and control of them as one of the key emergency works. In real life, multiple network public opinion emergencies may break out in a certain place at the same time. So, emergency decision experts in multiple fields are necessarily invited for timely evaluating the comprehensive crisis of the online public opinion, and then limited emergency resources are used to give priority to respond to the one with the highest crisis [4]. Due to the complexity of network public opinion emergencies and the limited cognition of people, most of the decision problems for network public opinion emergencies are highly uncertain. It is hard for decision makers to provide precise assessments in the assessment process [5-7]. Also, prior to the selection of the highest crisis, it is preferable that experts reach a high degree of consensus among their assessments or opinions $[8,9]$. Therefore, in essence, the selection of the network public opinion emergency with the highest crisis is a consensus-driven multiattribute group decision making (MAGDM) with highly uncertain information.

MAGDM can be defined as a decision situation where multiple decision makers or experts evaluate two or more possible alternatives according to multiple attributes. In realworld MAGDM, the increasing complexity and uncertainties of decision problems drive the experts to consider complex linguistic expressions instead of single linguistic terms [10]. In the last decade, some studies have successively attempted to model and compute with specific types of complex linguistic expressions. For example, by combining the merits of the hesitant fuzzy set (HFS) and linguistic terms, Rodriguez et al. introduced [11] hesitant fuzzy linguistic term set (HFLTS) that is characterized by a set of consecutive linguistic terms to increase the richness and flexibility of linguistic information elicitation using linguistic expressions. Considering that linguistic terms involved in an expression derived by the group may be not always consecutive, Wang [12] proposed extended HFLTS (EHFLTS). Inspired by the proportional 2-tuple linguistic representation model (provided by Wang and Hao [13]), Zhang et al. [14] initially developed linguistic distribution assessment (LDA). Compared with HFLTS, the characteristic of LDA is that symbolic proportions are assigned to all linguistic terms of a given linguistic term set. Following that initiative, the concept of possibility distribution for HFLTS (PDHFLTS) was developed by $\mathrm{Wu}$ and $\mathrm{Xu}$ [15] to model weight assignment for several discrete linguistic terms, where an HTLFS is ideally assumed to be uniformly distributed on its ordered linguistic terms [16, 17]. Further, under group decision-making (GDM) environments, the hesitant linguistic assessments of experts and the proportional information of each generalized linguistic term may need to be simultaneously considered [18, 19]. For instance, in terms of a questionnaire about the hazard degree of an emergency, 15 respondents (out of 100) stated it is "very high," 70 respondents stated it is "high," 10 respondents believed it is "slightly high," and others did not say anything. The collective assessment information can be denoted as $\{($ very high, 0.15), (high, 0.7), (slightly high, 0.1)\}. For this purpose, Chen et al. [20] initially proposed proportional HFLTS (PHFLTS) to simulate this decision scenario. Almost at the same time, Pang et al. [21] introduced probabilistic linguistic term set (PLTS) with a different name for the similar idea. Here, it needs to be emphasized that PDHFLTS, PHFLTS, and PLTS as variants of LDA are designed for different purposes. However, they are essentially derived from the idea of LDA and are even mathematically consistent with LDA in some sense. The taxonomy of the existing distributed linguistic representations and their comparison results can be found in a recent overview provided by $\mathrm{Wu}$ et al. [22]. In the last few years, PLTS, as a type of distributed linguistic representations, has occupied a dominant place in recent development and advancement of the linguistic decision-making community due to the following two possible reasons: (a) one is a concise and clear mathematical form and (b) the other is the focus and citation from related well-known scholars. In particular, MAGDM problems with PLTSs have received considerable attention. Related studies mainly concentrate on aggregation operators, comparison rules, measures, and decision-making methods [23].

Traditionally, only a selection process on all possible alternatives, which is composed of two phases: aggregation and exploitation, is applied to solving GDM problems. However, for many real-world problems closely related to citizens such as raising taxes, infrastructure constructions, and emergency responses, the resolution scheme obtained only by the selection process may not be supported by all or most experts since differences of individual opinions have not been fully taken into account. To do so, consensus reaching process (CRP) was introduced as an additional phase in the resolution of GDM problems [24, 25]. In general, consensus is defined as a dynamic and interactive group decision process coordinated by a human figure that is known as moderator (either real or virtual). Most classical consensus building is based on the interactive CRPs with the two classic consensus rules: identification rule (IR) and direction rule (DR) [26, 27]. Until now, classical CRPs have been extended and improved in different complex structures and contexts (e.g., fuzzy context [28], heterogeneous and uncertain preferences [29, 30], large-scale group [31], social network [32], dynamic environments [33], and minimum cost consensus [34]). In particular, for consensus-driven MAGDM with PLDMs, Wu and Liao [35] developed a consensus-based PL-GLDS method. However, to the best of our knowledge, the CRPs for MAGDM with PLDMs are not rich enough because most of the relevant studies in the probabilistic linguistic environment are based on probabilistic linguistic preference relations (PLPRs). Fortunately, a wealth of existing CRPs in other different structures and 
contexts mentioned above can be utilized to obtain some inspiration in exploring the novel PLDMs-based CRPs.

Consensus measures can usually be required to conduct the consensus checking and guide consensus repairing in CRPs [36]. In consensus-driven probabilistic linguistic MAGDM, we need to accurately measure the consensus degree between the individuals and collective opinions. Distance measures are natural to depict the deviations or differences between experts' opinions, which have been widely applied to the consensus measure in GDM. Recently, by turning to linguistic scale functions, the existing distances between PLTSs have been improved, which greatly promote the further development of consensus measures with PLTSs. For example, utilizing equivalent information of LTSs instead of their subscripts, Mao et al. [37] put forward a new Euclidean distance. Two novel distance measures (i.e., a generalized relative distance and an extended Hausdorff distance) for PLTSs were defined by Wang et al. [38]. Besides, considering that both Pang et al.'s distance measures [21] and Zhang et al.'s distance measures [39] fail to represent the differences between probabilities and the differences between linguistic terms, simultaneously, $\mathrm{Wu}$ et al. [40] established a new distance measure of PLTSs after introducing an adjustment method to derive the same probability set of pairwise PLTSs. Further, Wu and Liao's distance measure of the PLTSs has been improved by incorporating the unbalanced scenario [35], which is employed to set up the correlation coefficient between two PLTSs and then to measure the consensus degree between the individuals and collective opinions.

To design a suitable consensus reaching mechanism for the specific problem is another focus of CRPs. Generally, according to the way to guide the discussion process, the CRPs can be divided into two categories: feedback versus no feedback [28]. The feedback mechanisms are usually supervised by a moderator. The moderator, who plays a central role in the decision making, provides the experts with feedback and advice so that they can move towards a predefined consensus level [41]. Whether or not to modify the individual preferences needs to be determined by the experts. On the contrary, the no-feedback mechanisms tend to automatically update the preferences and/or weights of those experts who contribute the least to the group consensus, thereby reducing the need for a moderator. The CRPs without feedback mechanisms, which can avoid rigid corrections for individual preferences and thus make the experts' human intervention as minimal as possible, are very important and useful in certain special decision scenarios (such as emergency consensus decision making [42]). Some adaptive CRPs without feedback mechanism have been proposed and favored by scholars. Among them, the main adaptive consensus strategies include (a) generating recommendations by considering dynamic parameters such as minimum cost, consensus level, and adoption level $[34,43,44]$ and (b) updating expert weights based on individual consensus contributions $[45,46]$, non-cooperative behavior [47, 48], and self-confidence [49].

Based on the review conducted above, it is clear that the consensus-driven MAGDM with PLTSs has recently become a popular topic and the exploration of this issue has just begun. Moreover, some potential challenges can be identified as follows.

(1) Consensus measure in CRPs in different structures and contexts is often built based on different forms of distance. Nevertheless, the existing distance measures between pairwise PLTSs still have a few defects (see Section 4.1 for details). Although Wu and Liao [50] have already developed an effective distance measure of PLTSs from a new perspective, the computational complexity of the distance measure, originating from adjusting the probability set of any two PLTSs to be the same before calculating the distance, may have a major impact on its widespread application. For that reason, it is a leading challenge on how to balance between efficiency and accuracy in establishing a new distance measure of PLTSs, which will be utilized to construct a corresponding PLTSs-based consensus measure.

(2) In consensus-driven probabilistic linguistic MAGDM, when some individual assessments with nonconsensus occur, sometimes the moderator may recommend the identified individuals or experts to accept hard adjustment advice so that individuals can adjust their assessments for quickly reaching a certain consensus level. However, due to the double pressure of timeliness and reliability especially when confronted with some special decision-making environments (e.g., emergency consensus decision), the identified individuals or experts may be reluctant to or difficult to correct their assessments, to a certain extent, which may result in the interruption of the traditional CRPs. Given this defect of the traditional feedback mechanisms in actual execution, we urgently need to design a novel adaptive consensus reaching mechanism for probabilistic linguistic MAGDM.

(3) Until now, most of the CRPs under probabilistic linguistic environment are designed based on PLPRs rather than PLDMs. The possible cause is that a wealth of CPRs with crisp, fuzzy, or linguistic preference relations boosts the improvement of PLPRs-based CRPs. Note that multiple attributes with PLTSs are typically implied when experts evaluate alternatives under probabilistic linguistic environment. Therefore, it is theoretically and practically significant to explore a new MAGDM approach integrated with a PLDMs-based CRP. Besides, only one adaptive consensus improving technique (i.e., either generating recommendations or updating weights) is involved in the existing PLDMs-based adaptive CRPs, which may be not beneficial for quickly and comprehensively improving the consensus efficiency [51]. Accordingly, it is also necessary to integrate various adaptive consensus improving strategies into PLDMs-based CRPs. 
Motivated by these challenges, this paper aims to propose a novel consensus reaching model for MAGDM problems with probabilistic linguistic assessment information. The main innovations and contributions of this paper are as follows:

(1) We propose a PLTSs-based Wasserstein (called PLWasserstein) distance measure and show that the novel distance measure has been improved in both rationality and efficiency (see Section 4.2 for details).

(2) We construct an optimization model for objectively determining attribute weights, where the new Wasserstein distance measure between PLTSs is combined with the classical CCSD idea.

(3) We develop the individual cumulative consensus contribution (ICCC) measure and the group consensus measure, respectively. The former is used to punish the weights of experts that contribute the least to the group as well as to identify the individual assessment value with a maximum distance from the group opinion. The latter is utilized to guide the consensus process.

(4) We put forward a novel adaptive CRP for probabilistic linguistic MAGDM, where two adaptive consensus improving strategies (i.e., weight-updating and assessment-adjusting) are integrated. Subsequently, to obtain a consensus ranking of alternatives, we present a probabilistic linguistic MAGDM approach with the novel CRP.

(5) We make some comparative analyses with other MAGDM approaches to illustrate the effectiveness of the proposed approach by a case study concerning the evaluation of network public opinion emergencies.

The remainder of the paper is organized as follows. Section 2 reviews some related concepts and definitions. In Section 3, we describe consensus-driven MAGDM problems with probabilistic linguistic assessment information. A PLWasserstein distance measure is developed in Section 4. In Section 5, a novel adaptive consensus reaching model for probabilistic linguistic MAGDM is presented. The proposed approach is illustrated using a case of real network public opinion crisis rating evaluation, and we compare some similar methods in Section 6. Section 7 ends the paper with some concluding remarks.

\section{Preliminaries}

2.1. Probabilistic Linguistic Term Sets (PLTSs). In this section, the concepts and operational laws about linguistic term sets (LTSs) and probabilistic linguistic term sets (PLTSs) are given.

Definition 1 (see [52]). Let $S=\left\{s_{\alpha} \mid \alpha=-\tau, \ldots,-1,0,1, \ldots, \tau\right\}$ be a subscript-symmetric ordinal LTS, where $\tau$ is a positive integer, $s_{0}$ represents the assessment of "indifference" or "medium," and the remaining linguistic terms are distributed around $s_{0}$ in ascending order of intensity. $s_{i}$ satisfies the following conditions: (a) the set is ordered: $s_{\alpha} \leq s_{\beta}$ iff $\alpha \leq \beta$; (b) the negation operator is defined as neg $\left(s_{\alpha}\right)=s_{-\alpha}$.

For example, given an LTS $S$ with seven-point rating scales, then $S$ can be taken as

$S=\left\{s_{-3}=\right.$ "extremely bad," $s_{-2}=$ "very bad," $s_{-1}=$ "bad," $s_{0}=$ "medium," $s_{1}=$ "good," $s_{2}=$ "very good," $s_{3}=$ "extremely good" $\}$

Remark 1. To preserve all the given information, Xu extends the discrete LTS $S$ to a continuous linguistic term set $\bar{S}=\left\{s_{\alpha} \mid \alpha \in[-q, q]\right\}$, where $q(q \geq \tau)$ is a sufficiently large positive integer. Moreover, different types of LTSs, including balanced and unbalanced semantics, act as the different evaluation scales. Thus, an appropriate LTS needs to be chosen to evaluate qualitative variables according to practical issues.

Definition 2 (see [53]). Let $S=\left\{s_{\alpha} \mid \alpha=-\tau, \ldots,-1,0,1, \ldots, \tau\right\}$ be an LTS; the linguistic scale function $f$ of LTS $S$ can be defined by using a monotonically increasing function as

$$
f:\left[s_{-\tau}, s_{\tau}\right] \longrightarrow[0,1], \quad s_{\alpha} \longrightarrow \delta .
$$

Additionally, the equivalent linguistic term $s_{\alpha}$ of the membership degree $\delta$ is obtained by the following inverse function $f^{-1}$ :

$$
f^{-1}:[0,1] \longrightarrow\left[s_{-\tau}, s_{\tau}\right], \quad \delta \longrightarrow s_{\alpha} .
$$

For different types of LTSs, different linguistic scale functions can be designed to calculate the semantics of linguistic terms according to practical problems [35].

Especially, if the semantics of LTS $S$ are evenly distributed, then the linguistic scale function $f$ and its inverse function $f^{-1}$ can be formulated as

$$
\left\{\begin{array}{l}
f\left(s_{\alpha}\right)=\frac{\alpha+\tau}{2 \tau}=\delta, \\
f^{-1}(\delta)=s_{(2 \delta-1) \tau}=s_{\alpha} .
\end{array}\right.
$$


If the semantics are unevenly distributed and the semantic deviations between two adjacent linguistic terms on one side of the "medium" are increasing, then

$$
\left\{\begin{array}{l}
f\left(s_{\alpha}\right)=\frac{\gamma^{\tau}-\gamma^{-\alpha}}{2 \gamma^{\tau}-2} \times 1_{\{\alpha \in[-\tau, 0]\}}+\frac{\zeta^{\tau}+\zeta^{\alpha}-2}{2 \zeta^{\tau}-2} \times 1_{\{\alpha \in[0, \tau]\}}=\delta, \\
f^{-1}(\delta)=s_{-\log _{\gamma}\left(\gamma^{\tau}-\left(2 \gamma^{\tau}-2\right) \delta\right) \times 1_{\{\alpha \in[-\tau, 0]\}}+\log _{\zeta}\left(\left(2 \zeta^{\tau}-2\right) \delta-\zeta^{\tau}+2\right) \times 1_{\{\alpha \in[0, \tau]\}}}=s_{\alpha},
\end{array}\right.
$$

where $1_{\{\alpha \in[-\tau, 0]\}}$ and $1_{\{\alpha \in[0, \tau]\}}$ are both indicative functions, and two parameters $\gamma$ and $\zeta$ satisfy $\gamma>1, \zeta>1$.

Definition 3 (see [21]). Let $S=\left\{s_{\alpha} \mid \alpha=-\tau, \ldots,-1,0,1\right.$, $\ldots, \tau\}$ be a subscript-symmetric ordinal LTS. A PLTS on $S$ can be expressed as

$$
L(p)=\left\{L^{(k)}\left(p^{(k)}\right) \mid L^{(k)} \in S, \quad p^{(k)} \geq 0, k=1,2, \ldots, \# L(p), \quad \sum_{k=1}^{\# L(p)} p^{(k)} \leq 1\right\}
$$

where $L^{(k)}\left(p^{(k)}\right)$ denotes the $k$-th linguistic term $L^{(k)}$ with the probability $p^{(k)}$ (the meaning of probability can be the weight, the possibility degree, or the trust degree) and $\# L(p)$ is the number of all different linguistic terms in $L(p)$. Moreover, the expression $\sum_{k=1}^{\# L(p)} p^{(k)}<1$ indicates that the DM, due to his/her lack of knowledge, cannot provide complete assessment information, resulting in the absence of partial probability information.
Some basic operations of PLTS have been reviewed in Ref. [23].

Definition 4 (see [21]). Given a PLTS $L(p)=\left\{L^{(k)}\left(p^{(k)}\right) \mid k=\right.$ $1,2, \ldots, \# L(p)\}$ with $\sum_{k=1}^{\# L(p)} p^{(k)}<1$, the normalized PLTS $\widehat{L}(p)$ is defined as

$$
\widehat{L}(p)=\left\{L^{(k)}\left(\widehat{p}^{(k)}\right) \mid L^{(k)} \in S, \quad \widehat{p}^{(k)} \geq 0, k=1,2, \ldots, \# \widehat{L}(p), \quad \sum_{k=1}^{\# L(p)} \widehat{p}^{(k)}=1\right\}
$$

where $\widehat{p}^{(k)}=\left(p^{(k)} / \sum_{k=1}^{\left.\# \widehat{L}(p) p^{(k)}\right)}\right.$ for all $k=1,2, \ldots, \# \widehat{L}(p)$.

Definition 5 (see [21]). Given two PLTSs on $S L_{i}(p)=\left\{L_{i}^{(k)}\right.$ $\left.\left(p_{i}^{(k)}\right) \mid k=1,2, \ldots, \# L_{i}(p)\right\}, \quad i=1,2$, where $\# L_{i}(p)$ is the number of all different linguistic terms in $L_{i}(p)$, if $\# L_{1}(p) \neq$ $\# L_{2}(p)$, when $\# L_{1}(p)>\# L_{2}(p)$, then we can add $\# L_{1}(p)-$ $\# L_{2}(p)$ linguistic terms to $L_{2}(p)$ so that the numbers of linguistic terms in $L_{1}(p)$ and $L_{2}(p)$ are identical. The added linguistic terms are supposed to be the smallest one in $L_{2}(p)$ and their corresponding probabilities are zero.

Definition 6 (see [39]). Given a PLTS $L(p)=\left\{L^{(k)}\left(p^{(k)}\right) \mid k=\right.$ $1,2, \ldots, \# L(p)\}$ on $S, r^{(k)}$ is the subscript of the linguistic term $L^{(k)}$.

(1) If all elements in the PLTSs are with different values of $r^{(k)} p^{(k)}$, then all the elements are arranged according to the values of $r^{(k)} p^{(k)}$ directly.

(2) If there are two or more elements with equal values of $r^{(k)} p^{(k)}$, then (a) when the subscripts $r^{(k)}$ $(k=1,2, \ldots, \# L(p))$ are unequal, $r^{(k)} p^{(k)}$ $(k=1,2, \ldots, \# L(p))$ are arranged according to the values of $r^{(k)}(k=1,2, \ldots, \# L(p))$ in ascending order; (b) when the subscripts $r^{(k)}(k=1,2, \ldots, \# L(p))$ are equal or incomparable, $r^{(k)} p^{(k)}$ $(k=1,2, \ldots, \# L(p))$ are arranged according to the values of $p^{(k)}(k=1,2, \ldots, \# L(p))$ in ascending order.

Similarly, we can obtain a descending-order PLTS.

Definition 7 (see $[35,40])$. Let $L_{i}(p)=\left\{L_{i}^{(k)}\left(p_{i}^{(k)}\right) \mid k=1,2\right.$, $\left.\ldots, \# L_{i}(p)\right\}, i=1,2$ be two normalized PLTSs on $S$ with a linguistic scale function $f$, where $L_{i}^{(k)}$ is the $k$-th LTS and $p_{i}^{(k)}$ is its corresponding probability in $L_{i}(p)$. Then, the general probabilistic linguistic expected value of PLTS $(E(L(p)))$ and the general variance value of PLTS $(\sigma(L(p)))$ are defined as follows, respectively.

$$
\begin{aligned}
E\left(L_{i}(p)\right) & =\sum_{k=1}^{\# L_{i}(p)} f\left(L_{i}^{(k)}\right) \cdot p_{i}^{(k)}, \\
\sigma\left(L_{i}(p)\right) & =\left(\sum_{k=1}^{\# L_{i}(p)}\left(f\left(L_{i}^{(k)}\right)-E\left(L_{i}(p)\right)\right)^{2} \cdot p_{i}^{(k)}\right)^{1 / 2} .
\end{aligned}
$$

For two PLTSs $L_{1}(p)$ and $L_{2}(p)$, if $E\left(L_{1}(p)\right)>$ $E\left(L_{2}(p)\right)$, then $L_{1}(p)>L_{2}(p)$. If $E\left(L_{1}(p)\right)=E\left(L_{2}(p)\right)$, then $\sigma\left(L_{1}(p)\right)<\sigma\left(L_{2}(p)\right), \quad L_{1}(p)>L_{2}(p) ; \quad$ if $\quad E\left(L_{1}(p)\right)=$ $E\left(L_{2}(p)\right)$, then $\sigma\left(L_{1}(p)\right)=\sigma\left(L_{2}(p)\right), L_{1}(p) \sim L_{2}(p)$. 
Definition 8. (see $[35,50])$ Let $E=\left\{e_{1}, e_{2}, \ldots, e_{h}\right\}(h \geq 2)$ be a set of $h$ experts whose expert vector is $\lambda=\left(\lambda^{(1)}, \lambda^{(2)}, \ldots, \lambda^{(h)}\right)^{T}$ such that $\lambda^{(k)} \in[0,1]$ and $\sum_{k=1}^{h} \lambda^{(k)}=1$. Suppose that

$$
L^{(k)}(p)=\left\{L^{(l(k))}\left(p^{(l(k))}\right) \mid L^{(l(k))} \in S, p^{(l(k))} \geq 0, l(k)=1,2, \ldots, \# L^{(k)}(p), \quad \sum_{l(k)=1}^{\# L^{(k)}(p)} p^{(l(k))} \leq 1\right\}, \quad k=1,2, \ldots, \tau
$$

are PLTSs on an LTS $S$ provided by $\tau$ experts while the remaining $h-\tau$ experts do not give any evaluation. Then, we can express the collective evaluation by using the following PLTS:

$$
L^{(G)}(p)=\left\{L^{(l)}\left(p^{(l)}\right) \mid L^{(l)} \in S, p^{(l)}=\sum_{k=1}^{\tau} \lambda^{(k)} v^{(l(k))}, l=1,2, \ldots, \# L^{(G)}(p)\right\}
$$

where $v^{(l(k))}$ is the weight of $L^{(l(k))}$ in $L^{(k)}(p)$ and $v^{(l(k))}=\left\{\begin{array}{ll}p^{(l(k))}, & \text { if } L^{(l(k))} \in L^{(k)}(p) \\ 0, & \text { if } L^{(l(k))} \notin L^{(k)}(p)\end{array}\right.$.

If the weights of experts are the same or not given, we can suppose that $\lambda^{(k)}=(1 / h), k=1,2, \ldots, h$.

\subsection{Consensus-Driven MAGDM Framework. In a classical} MAGDM, there is a discrete set of alternatives, a set of predefined attributes, and a group of two or more experts (characterized by their backgrounds and knowledge) who express their opinions or preferences about the set of alternatives under the set of predefined attributes. Clearly, in any consensus-driven MAGDM, the experts should reach an acceptable degree of group consensus on the solution set of alternatives [34]. To do so, a classical consensus MAGDM usually consists of two basic components: consensus process and selection process [25].

2.2.1. Consensus Process. This process refers to how to obtain the maximum degree of agreement among the experts on the solution alternatives. Usually, before the selection process, it is carried out to achieve a solution to the GDM problems which can be accepted by all experts in the group. Generally, to avoid absolute "winners" and "losers" in a group, a dynamic and iterative discussion procedure is indispensable so that experts can reach a certain degree of consensus in this process. Three main phases involved in this process include (a) measuring level of group consensus, (b) modifying evaluations or updating decision weight, and (c) generating feedback information, which can be required for conducting CRPs. An overall scheme of the different phases carried out in a consensus process is depicted in Figure 1.

2.2.2. Selection Process. This process represents how to obtain the solution set of alternatives from the opinions on the alternatives given by the experts. It is composed of two phases: aggregation and exploitation. A collective evaluation based on the opinions expressed by the experts is defined in the aggregation phase. The collective evaluation is transformed into a global ranking by the exploitation phase. A general scheme of the two phases performed in a selection process is shown in Figure 2.

2.3. Wasserstein Metric. The Wasserstein metric or distance is a distance function defined between probability distributions on a given metric space, also called (Monge-) Kantorovich(-Rubinstein) distance that can be traced back to the early research of Monge-Kantorovich optimal transport problem [54]. The terminology Wasserstein distance became popular, mainly in Western literature, following Dobrushin who studied some of its topological properties and referred to an earlier work by Wasserstein [55]. The long history of Wasserstein distance can be found in the literature [56] for more details.

There are a host of metrics available to quantify the distance between probability measures; some are not even metrics in the strict sense of the word but are simply notions of "distance" that have been proven useful to consider [57]. How does one choose among all these metrics? Issues that can affect a metric's desirability include whether it has an interpretation applicable to the problem at hand, important theoretical properties, or useful bounding techniques [58]. Kantorovich and Rubinstein [59] first recognized Wasserstein metric as a reasonable distance on spaces of random variables or probability distributions. In Ref. [59], to measure the distance of probability distributions, an infinite-dimensional linear programming is taken into consideration, which is inspired by the problem of optimal mass transportation. Roughly speaking, the minimal effort required to reconfigure the probability mass of one distribution to recover the other distribution was measured by the Wasserstein metric.

We briefly review the definition and related properties of the Wasserstein metric in the following.

Definition 9 (see $[56,57])$. Let $(\mathscr{X}, d)$ be a Polish (i.e., complete, separable, and metric) space, and let $q \in[1,+\infty)$. For any two Borel probability measures $\mu$ and $\nu$ on $\mathscr{X}$, the $q$ order Wasserstein distance between $\mu$ and $\nu$ is defined as 


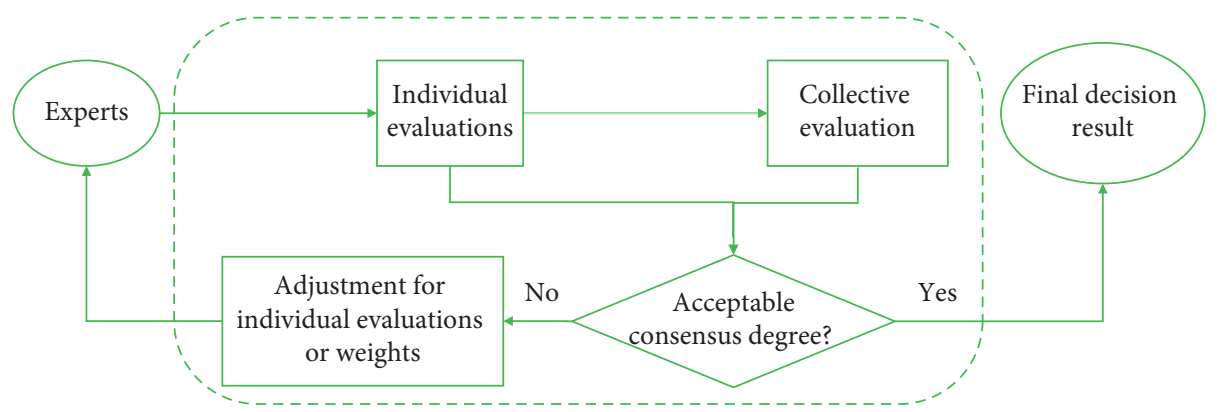

FIGURE 1: The consensus reaching process.

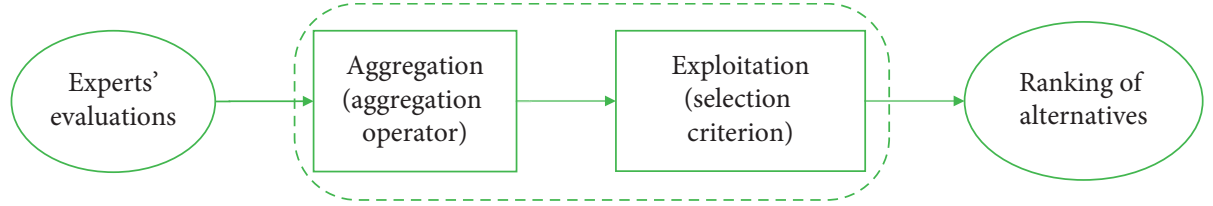

FIGURE 2: The selection process in MAGDM problems.

$$
\mathrm{WD}_{q}^{d}(\mu, \nu)=\left(\inf _{\gamma \in \Gamma(\mu, \nu)} \int_{X \times X} \mathrm{~d}(x, y)^{q} \mathrm{~d} \gamma(x, y)\right)^{1 / q}=\inf _{\substack{\gamma \in \Gamma(\mu, \nu) \\ X \sim \mu, Y \sim \nu}}\left\{\left[\mathbb{E}_{\gamma} d(X, Y)^{q}\right]^{1 / q}\right\},
$$

where $\Gamma(\mu, \nu)$ denotes the set of all couplings of $\mu$ and $\nu$ on $\mathscr{X} \times \mathscr{X}$, that is, the set of all joint probability measures over $\mathscr{X} \times \mathscr{X}$ whose marginals are $\mu$ and $\nu$, satisfying $\gamma(A \times \mathscr{X})=$ $\mu(A)$ and $\gamma(\mathscr{X} \times B)=\nu(B)$ for all measurable subsets $A, B \subset \mathscr{X}$. Here, the infimum is taken over all probability measures $\gamma(\in \in \Gamma(\mu, \nu))$ with prescribed marginals $\mu$ and $\nu$, that is, all pairs of random vectors $X$ and $Y$ marginally distributed as $\mu$ and $\nu$, respectively.

Remark 2 (see $[60,61])$. The 1-order Wasserstein distance $\mathrm{WD}_{1}^{d}$ (in the case $q=1$ ) is also descriptively called the earth mover's distance (EMD), which measures the least amount of work required to move a pile of soil from one shape to another. Here, a unit of work corresponds to transporting a unit of soil by a unit of ground distance.

Remark 3 (see [62]). Compared to the K-L divergence and the J-S divergence, the outstanding advantage of the Wasserstein distance is that the difference between the two probability distributions can still be reflected even if the support sets of the two distributions do not overlap or the overlap is very small. However, in this case, the J-S divergence is constant while the $\mathrm{K}-\mathrm{L}$ divergence may be meaningless.

\section{Consensus-Driven MAGDM Problems with Probabilistic Linguistic Assessment Information}

This paper mainly investigates consensus-driven MAGDM problems with probabilistic linguistic assessment information. The characteristics of assessment information provided by experts are mainly reflected in the following two aspects. On the one hand, the experts are accustomed to expressing their assessments or preferences over the considered alternatives in the form of linguistic terms like "good," "fair," or "poor." On the other hand, the experts may not only hesitate between more than one linguistic term but also prefer some of the possible linguistic terms so that the hesitant linguistic terms may have different importance degrees, such as " $40 \%$ sure it is good and $60 \%$ sure it is fair." This kind of problem usually occurs when evaluating hotels on TripAdvisor.com, emergency rescue plans, intelligent medical hospitals, and sustainable suppliers.

Let $S=\left\{s_{\alpha} \mid \alpha=-\tau, \ldots,-1,0,1, \ldots, \tau\right\}$ be a subscriptsymmetric LTS, $A=\left\{a_{1}, a_{2}, \ldots, a_{m}\right\}(m \geq 2)$ be a finite set of $m$ alternatives, $E=\left\{e_{1}, e_{2}, \ldots, e_{h}\right\}(h \geq 2)$ be a set of $h$ experts, and $C=\left\{c_{1}, c_{2}, \ldots, c_{n}\right\}=C^{\text {benefit }} \cup C^{\text {cost }}(n \geq 2)$ be a set of $n$ attributes, where $C^{\text {benefit }}$ and $C^{\text {cost }}$ represent the benefit attribute and cost attribute sets, respectively. The assessment value given by expert $e_{k}$ on alternative $a_{i}$ with respect to attribute $c_{j}$ is denoted as $r_{i j}^{(k)}$ in the form of a PLTS $L_{i j}^{(k)}(p)=$ $\left\{L_{i j}^{(l(k))}\left(p_{i j}^{(l(k))}\right) \mid L_{i j}^{(l(k))} \in S, p_{i j}^{(l(k))} \geq 0, l(k)=1,2, \ldots, \# L_{i j}^{(k)}(p)\right.$, $\left.\sum_{l(k)=1}^{\# L_{i j}^{(k)}(p)} p_{i j}^{(l(k))} \leq 1\right\} \quad(i=1,2, \ldots, m ; j=1,2, \ldots, n ; k=1,2, \ldots$, h). $R^{(k)}=\left(r_{i j}^{(k)}\right)_{m \times n}$ is called a probabilistic linguistic decision matrix (PLDM) provided by expert $e_{k}$ on $m$ alternatives under $n$ attributes. Additionally, the weight vector over experts $E$ is denoted as $\lambda=\left(\lambda^{(1)}, \lambda^{(2)}, \ldots, \lambda^{(h)}\right)^{T}$ such that $\lambda^{(k)} \in[0,1]$ and $\sum_{k=1}^{h} \lambda^{(k)}=1 . w=\left(w^{(1)}, w^{(2)}, \ldots, w^{(n)}\right)^{T}$ belonging to $\Omega$ is the attribute weight vector, satisfying $w^{(j)} \in[0,1]$ and $\sum_{j=1}^{n} w^{(j)}=1$, where $\Omega$ represents an incomplete attribute weight information set. 
The main problem to be solved in this paper is to establish a consensus reaching model for probabilistic linguistic MAGDM problems with PLDMs. The following questions arise when investigating this research problem. (a) How do we quantify the difference between any two PLTSs accurately and efficiently after recognizing the shortcomings of existing measure methods? (b) How do we fully mine potential attribute weight information to serve the consensual sorting or selection of alternatives after collecting the PLDMs information? (c) How do we design a fast, valid, and adaptive CRP to reach a certain degree of consensus in the group with PLDMs?

\section{PLTSs-Based Wasserstein Distance Measure}

In this section, assume that all PLTSs have been normalized by Definition 4. That is, any PLTS $L(p)$ satisfies $\sum_{k=1}^{\# L(p)} p^{(k)}=1$. Firstly, the existing distances between PLTSs are reviewed and some of their defects one by one through some specific examples are pointed out. Then, a new Wasserstein distance measure between PLTSs is proposed, and its properties are given.

4.1. Existing Distance Measures between PLTSs. Recently, linguistic scale functions (see Definition 2) have been integrated into PLTSs-based distance measures. Related definitions of distance measures are as follows.

Definition 10 (see [37]). Let $S=\left\{s_{\alpha} \mid \alpha=-\tau, \ldots,-1,0,1\right.$, $\ldots, \tau\}$ be an LTS with the linguistic scale function $f$ and $L_{i}(p)=\left\{L_{i}^{(k)}\left(p_{i}^{(k)}\right) \mid k=1,2, \ldots, \# L_{i}(p)\right\}, i=1,2$ be two ascending-ordered normalized PLTSs on $S$ with $\# L_{1}(p)=$ $\# L_{2}(p)$ (see Definition 6). Then, the Euclidean distance between $L_{1}(p)$ and $L_{2}(p)$ is defined as follows.

$$
D_{E}\left(L_{1}(p), L_{2}(p)\right)=\left(\sum_{k=1}^{\# L_{1}(p)} \frac{\left(f\left(L_{1}^{(k)}\right) \cdot p_{1}^{(k)}-f\left(L_{2}^{(k)}\right) \cdot p_{2}^{(k)}\right)^{2}}{\# L_{1}(p)}\right)^{1 / 2} .
$$

Example 1. Consider two ascending-ordered normalized PLTSs $L_{1}(p)=\left\{s_{1}(1)\right\}=\left\{s_{1}(0), s_{1}(1)\right\}$ and $L_{2}(p)=\left\{s_{-}\right.$ $\left.3(0.2), s_{2}(0.8)\right\}$ based on $S=\left\{s_{-3}, \ldots, s_{-1}, s_{0}, s_{1}, \ldots, s_{3}\right\}$. For the sake of simplicity, suppose that the linguistic scale function $f$ is as shown in equation (4). By equation (13), it is easy to get $D_{E}\left(L_{1}(p), L_{2}(p)\right)=0$. Obviously, the two PLTSs are not completely identical. Thus, the Euclidean distance obtained by using equation (13) is more or less irrational.

Definition 11 (see [38]). Let $S=\left\{s_{\alpha} \mid \alpha=-\tau, \ldots,-1,0,1, \ldots, \tau\right\}$ be an LTS with the linguistic scale function $f$. Two normalized PLTSs $L_{1}(p)=\left\{L_{1}^{(k)}\left(p_{1}^{(k)}\right) \mid k=1,2, \ldots, \# L_{1}(p)\right\}$ and $L_{2}(p)=\left\{L_{2}^{(l)}\left(p_{2}^{(l)}\right) \mid l=1,2, \ldots, \# L_{2}(p)\right\}$ on $S$ are given, whose elements are arranged according to the value of $L_{1}^{(k)}$ $\left(k=1,2, \ldots, \# L_{1}(p)\right)$ and $L_{2}^{(l)} \quad\left(l=1,2, \ldots, \# L_{2}(p)\right)$ in descending order, respectively. The generalized relative distance $D_{G}$ between $L_{1}(p)$ and $L_{2}(p)$ and the extended Hausdorff distance $D_{H}$ between $L_{1}(p)$ and $L_{2}(p)$ are defined as follows:

$$
\begin{aligned}
& D_{G}\left(L_{1}(p), L_{2}(p)\right)=\left|\left(\sum_{k=1}^{\# L_{1}(p)}\left|f\left(s_{\tau}\right)-f\left(L_{1}^{(k)}\right)\right|^{q} \cdot p_{1}^{(k)}\right)^{1 / q}-\left(\sum_{l=1}^{\# L_{2}(p)}\left|f\left(s_{\tau}\right)-f\left(L_{2}^{(l)}\right)\right|^{q} \cdot p_{2}^{(l)}\right)^{1 / q}\right|, \\
& D_{H}\left(L_{1}(p), L_{2}(p)\right)=\max _{k=1,2, \ldots, \# L_{1}(p)}\left\{\left(\frac{1}{2}\left(\min _{l=1,2, \ldots, \# L_{2}(p)}\left[\left|f\left(L_{1}^{(k)}\right)-f\left(L_{2}^{(l)}\right)\right|^{q}+\left|f\left(L_{1}^{(k)}\right) p_{1}^{(k)}-f\left(L_{2}^{(l)}\right) p_{2}^{(l)}\right|^{q}\right]\right)\right)^{1 / q}\right\} .
\end{aligned}
$$

Example 2. Consider two descending-ordered PLTSs $L_{1}(p)=\left\{s_{1}(1)\right\}$ and $L_{3}(p)=\left\{s_{2}(0.5), s_{0}(0.5)\right\}$ based on $S=\left\{s_{-3}, \ldots, s_{-1}, s_{0}, s_{1}, \ldots, s_{3}\right\}$. For the sake of simplicity, suppose that the linguistic scale function $f$ is as shown in equation (4). In particular, if $q=1$, it holds that $D_{G}\left(L_{1}(p), L_{3}(p)\right)=0$ by equation (14). So, $D_{G}$ does not satisfy the uniqueness axiom of distance. Additionally, through using equation (15), we can conclude that $D_{H}\left(L_{1}(p), L_{3}(p)\right)=(5 / 24) \quad$ while $\quad D_{H}\left(L_{3}(p), L_{1}(p)\right)=$ $(7 / 24)$. So, $D_{H}\left(L_{1}(p), L_{3}(p)\right) \neq D_{H}\left(L_{3}(p), L_{1}(p)\right)$, namely, $D_{H}$ does not satisfy the symmetry axiom of distance.

$\mathrm{Wu}$ and Liao [50] realized the fact that multiplying the probability by the subscript of the corresponding linguistic term of a PLTS or multiplying the probabilities of two PLTSs is unreasonable. By adjusting the PLTSs with the same probability set, they proposed three kinds of distance measures reflecting on the difference of linguistic terms and probabilities simultaneously. Furthermore, considering the linguistic scale functions of different linguistic terms in the PLTSs, Wu and Liao [35] developed the generalized distance measures between two PLTSs, which is shown as follows.

Definition 12 (see [35]). Let $S=\left\{s_{\alpha} \mid \alpha=-\tau, \ldots,-1,0,1, \ldots, \tau\right\}$ be an LTS with the linguistic scale function $f$. For two normalized PLTSs $L_{1}(p)=\left\{L_{1}^{(k)}\left(p_{1}^{(k)}\right) \mid k=1,2, \ldots, \# L_{1}(p)\right\}$ and $L_{2}(p)=\left\{L_{2}^{(l)}\left(p_{2}^{(l)}\right) \mid l=1,2, \ldots, \# L_{2}(p)\right\}$ on $S$, suppose that the adjusted forms are $L_{1}^{*}(p)=\left\{L_{1}^{*(k)}\left(p^{*(k)}\right) \mid k=1,2, \ldots\right.$, $K\}$ and $\left.L_{2}^{*}(p)=\left\{L_{2}^{*(k)}\left(p^{*(k}\right)\right) \mid k=1,2, \ldots, K\right\}$ with the same 
possibility set as $P=\left\{p^{*(1)}, p^{*(2)}, \ldots, p^{*(K)}\right\}$ (for details about the adjusting process, refer to Ref. [40]). The generalized distance $D_{A G}$ between $L_{1}(p)$ and $L_{2}(p)$ with the above adjustment strategy of PLTSs can be defined as

$$
D_{A G}\left(L_{1}(p), L_{2}(p)\right)=\left(\sum_{k=1}^{K}\left|f\left(L_{1}^{*(k)}\right)-f\left(L_{2}^{*(k)}\right)\right|^{q} \cdot p^{*(k)}\right)^{1 / q} .
$$

Example 3. (continued from Examples 1 and 2). In particular, take $q=1$. First, adjust the three PLTSs (i.e., $L_{1}(p)=\left\{s_{1}(1)\right\}, \quad L_{2}(p)=\left\{s_{-3}(0.2), s_{2}(0.8)\right\}$ and $L_{3}(p)=$ $\left.\left\{s_{2}(0.5), s_{0}(0.5)\right\}\right)$ with the same probability set $P=\{0.2,0.3,0.5\}$. Then, we can obtain three adjusted PLTSs: $L_{1}^{*}(p)=\left\{s_{1}(0.2), s_{1}(0.3), s_{1}(0.5)\right\}, \quad L_{2}^{*}(p)= \begin{cases}s_{-} & 3(0.2),\end{cases}$ $\left.s_{2}(0.3), s_{2}(0.5)\right\}$, and $L_{3}^{*}(p)=\left\{s_{0}(0.2), s_{0}(0.3), s_{2}(0.5)\right\}$. Finally, by equation (16), we can obtain $D_{A G}\left(L_{1}(p)\right.$, $\left.L_{2}(p)\right)=(4 / 15), D_{A G}\left(L_{1}(p), L_{3}(p)\right)=(1 / 6), D_{A G}\left(L_{2}(p)\right.$, $\left.L_{3}(p)\right)=(1 / 5)$.

Remark 4. The above generalized distance $D_{A G}$ satisfies the basic principles of distance measure [35], even including the property of triangle inequality. In fact, for any three normalized PLTSs $\left(L_{1}(p), L_{2}(p)\right.$, and $\left.L_{3}(p)\right)$, we can always adjust them with the same possibility set by using the adjustment strategy of PLTSs [40]. So, the triangle inequality (i.e., $\quad D_{A G}\left(L_{1}(p), L_{3}(p)\right) \leq D_{A G}\left(L_{1}(p), L_{2}(p)\right)+D_{A G}(L$ $\left.{ }_{2}(p), L_{3}(p)\right)$ can also be proven to be true in spite of missing specific elaboration in Refs. [35, 40]. However, it takes too much time to implement the adjustment strategy for PLTSs, which is not conducive to fast and effective PLTSs-based decision making.

4.2. PLTSs-Based Wasserstein Distance Measure. Given the shortcomings of the above definitions on distances between PLTSs, a Wasserstein distance measure between PLTSs is defined.

First, let $L(p)=\left\{L^{(k)}\left(p^{(k)}\right) \mid k=1,2, \ldots, \# L(p)\right\}$ be a normalized PLTS by Definition 4 , where $L^{(k)}$ is the $k$-th LTS $S$ and $p^{(k)}$ is its corresponding probability in the PLTS $L(p)$. Given the linguistic scale function $f$ of LTS $S$ (see Definition 2 ), any PLTS is considered as a discrete probability distribution. That is, the linguistic scale degree $X=f\left(L^{(k)}\right)$ of linguistic terms $L^{(k)}$ in the PLTS $L(p)$ can be assumed to be a random variable, which follows a discrete probability distribution (denoted by $\operatorname{DPD}(L(p), f)$ ), with the following probability mass function:

$$
X \sim D P D_{\text {PLTS }}(L(p), f): P\left(X=f\left(L^{(k)}\right)\right)=p^{(k)}, \quad k=1,2, \ldots, \# L(p) .
$$

Then, we define the following q-order Wasserstein distance measure between PLTSs, by resorting to the idea of classical Wasserstein distance (see Definition 9).

Definition 13. Suppose that $d(x, y): \mathbb{R}^{2} \longrightarrow \mathbb{R}$ is both a distance function and a binary Borel measurable function. Let $\quad q \in[1,+\infty)$ and $L_{i}(p)=\left\{L_{i}^{(k)}\left(p_{i}^{(k)}\right) \mid k=1,2\right.$, $\left.\ldots, \# L_{i}(p)\right\}, i=1,2$ be two normalized PLTSs by Definition 4 , where $L_{i}^{(k)}$ is the $k$-th LTS and $p_{i}^{(k)}$ is its corresponding probability in $L_{i}(p)$. The $q$-order Wasserstein distance measure between PLTSs can be defined as

$$
\operatorname{PLWD}_{q}^{d}\left(L_{1}(p), L_{2}(p)\right)=\inf _{\gamma}\left\{\left[\mathbb{E}_{\gamma} d\left(X_{1}, X_{2}\right)^{q}\right]^{1 / q}:\left(X_{1}, X_{2}\right) \sim \gamma, X_{1} \sim \mu_{1}, X_{2} \sim \mu_{2}\right\}
$$

Here, the meaning of formula (18) is that the infimum is applied to the set of all joint probability distributions $\gamma$ with prescribed marginals $\mu_{1}=\operatorname{DPD}_{\text {PLTS }}\left(L_{1}(p), f\right)$ and $\mu_{2}=$ $\operatorname{DPD}_{\text {PLTS }}\left(L_{2}(p), f\right)$, where $X_{i} \sim \mu_{i}$ indicates that the linguistic scale degree $X_{i}$ of linguistic terms $L_{i}^{(k)}$ follows the discrete probability distribution $\mu_{i}$ with the probability mass function $P\left(X_{i}=f\left(L_{i}^{(k)}\right)\right)=p_{i}^{(k)}, k=1,2, \ldots, \# L_{i}(p), i=1,2$.

Some properties of Definition 13 are given in the following.

Proposition 1. Let $S=\left\{s_{\alpha} \mid \alpha=-\tau, \ldots,-1,0,1, \ldots, \tau\right\}$ be an LTS with the linguistic scale function $f$. Suppose that $L_{1}(p)$, $L_{2}(p)$, and $L_{3}(p)$ are three normalized PLTSs on $S$. The $q-$ order PL-Wasserstein distance measure $P L W D_{q}^{d}$ satisfies the following basic principles of distance:

(1) $P L W D_{q}^{d}\left(L_{1}(p), L_{2}(p)\right) \geq 0$

(2) $P L W D_{q}^{d}\left(L_{1}(p), L_{2}(p)\right)=0$, if and only if $L_{1}(p) \stackrel{q}{=} L_{2}(p)$

(3) $P L W D_{q}^{d}\left(L_{1}(p), L_{2}(p)\right)=P L W D_{q}^{d}\left(L_{2}(p), L_{1}(p)\right)$
(4) $P L W D_{q}^{d}\left(L_{1}(p), L_{3}(p)\right) \leq P L W D_{q}^{d}\left(L_{1}(p), L_{2}(p)\right)+$ $P L W D_{q}^{d}\left(L_{2}(p), L_{3}(p)\right)$

Proof. (1) Since $d(x, y)$ is a distance function, then $d(x, y) \geq$ 0 . So, one has $\left[\mathbb{E}_{\gamma} d\left(X_{1}, X_{2}\right)^{q}\right]^{1 / q} \geq 0$. Thus, $\operatorname{PLWD}_{q}^{d}\left(L_{1}(p)\right.$, $\left.L_{2}(p)\right)=\inf _{\gamma}\left\{\left[\mathbb{E}_{\gamma} d\left(X_{1}, X_{2}\right)^{q}\right]^{1 / q}\right\} \geq 0$.

(2) On the one hand, if $L_{1}(p)=L_{2}(p)$, then $\operatorname{PLWD}_{q}^{d}$ $\left(L_{1}(p), L_{2}(p)\right)=0$ since $d\left(X_{1}, X_{2}\right)$ has a null diagonal, with the optimal coupling of $\left(X_{1}, X_{2}\right)$ such that $p^{(k l)}=0 ; p^{(k k)}$ $=p_{1}^{(k)}$, where $k=1,2, \ldots, \# L_{1}(p), l=1,2, \ldots, \# L_{2}(p)$. On the other hand, by the positivity of all off-diagonal elements of $\quad d\left(X_{1}, X_{2}\right), \quad \operatorname{PLWD}_{q}^{d}\left(L_{1}(p), L_{2}(p)\right)>0 \quad$ whenever $L_{1}(p) \neq L_{2}(p)$ (because in this case, an admissible coupling necessarily has a nonzero element outside the diagonal).

(3) Since $d(x, y)$ is a distance function, then the symmetry (i.e., $d(x, y)=d(y, x))$ remains true. So, it is clear that $\operatorname{PLWD}_{q}^{d}\left(L_{1}(p), L_{2}(p)\right)=\operatorname{PLWD}_{q}^{d}\left(L_{2}(p), L_{1}(p)\right)$.

(4) Let $\mu_{i}=\mathrm{DPD}_{\text {PLTS }}\left(L_{i}(p), f\right)(i=1,2,3)$ be three discrete probability distributions with the probability mass functions $P\left(X_{i}=f\left(L_{i}^{(k)}\right)\right)=p_{i}^{(k)}, \quad k=1,2, \ldots, \# L_{i}(p)$, 
$i=1,2,3$. Also, suppose that $\left(X_{1}, X_{2}\right)$ and $\left(Y_{1}, Y_{2}\right)$ are the optimal couplings of $\left(\mu_{1}, \mu_{2}\right)$ and $\left(\mu_{2}, \mu_{3}\right)$, respectively. Based on the Gluing Lemma [56], one can obtain tripe of random variables $\left(X_{1}^{\prime}, X_{2}^{\prime}, X_{3}^{\prime}\right)$, satisfying that $\left(X_{1}^{\prime}, X_{2}^{\prime}\right.$, ) has the same probability distribution as $\left(X_{1}, X_{2}\right)$ and $\left(X_{2}^{\prime}, X_{3}^{\prime}\right)$ has the same probability distribution as $\left(Y_{1}, Y_{2}\right)$. So, one can have

$$
\begin{aligned}
\operatorname{PLWD}_{q}^{d}\left(L_{1}(p), L_{3}(p)\right) & \leq\left[\mathbb{E}_{\gamma} d\left(X_{1}^{\prime}, X_{3}^{\prime}\right)^{q}\right]^{1 / q} \\
& \leq\left[\mathbb{E}_{\gamma}\left(d\left(X_{1}^{\prime}, X_{2}^{\prime}\right)+d\left(X_{2}^{\prime}, X_{3}^{\prime}\right)\right)^{q}\right]^{1 / q} \leq\left[\mathbb{E}_{\gamma} d\left(X_{1}^{\prime}, X_{2}^{\prime}\right)^{q}\right]^{1 / q}+\left[\mathbb{E}_{\gamma} d\left(X_{2}^{\prime}, X_{3}^{\prime}\right)^{q}\right]^{1 / q} \\
& =\operatorname{PLWD}_{q}^{d}\left(L_{1}(p), L_{2}(p)\right)+\operatorname{PLWD}_{q}^{d}\left(L_{2}(p), L_{3}(p)\right) .
\end{aligned}
$$

Here, the first inequality follows from the fact that $\left(X_{1}^{\prime}, X_{3}^{\prime}\right)$ is a coupling of $\left(\mu_{1}, \mu_{3}\right)$. The second employs the triangle inequality of the distance function $d(x, y)$. The third is an application of Minkowski inequality in $L^{q}(\mu)(q \geq 1)$. The last equality implies that $\left(X_{1}^{\prime}, X_{2}^{\prime}\right)$ and $\left(X_{2}^{\prime}, X_{3}^{\prime}\right)$ are the optimal couplings of $\left(\mu_{1}, \mu_{2}\right)$ and $\left(\mu_{2}, \mu_{3}\right)$, respectively.

Proposition 2. In particular, for $P L W D_{q}^{d}$, take $q=1$ and $a$ Hamming distance function $d(x, y)=|x-y|$ is given. The special 1-order PL-Wasserstein distance measure is denoted by PLWD $D_{1}^{\text {hd }}$. Besides the properties provided by Proposition 1, $P L W D_{1}^{\text {hd }}$ has the following properties:

(1) $0 \leq P L W D_{1}^{h d}\left(L_{1}(p), L_{2}(p)\right) \leq 1$

(2) $P L W D_{1}^{\text {hd }}\left(L_{1}(p), L_{2}(p)\right)=1$, if and only if either $L_{1}(p)=\left\{s_{\tau}(1)\right\} \quad$ and $L_{2}(p)=\left\{s_{-\tau}(1)\right\}$ or $L_{1}(p)=\left\{s_{-\tau}(1)\right\}$ and $L_{2}(p)=\left\{s_{\tau}(1)\right\}$

Proof. (1) Since the linguistic scale function $f$ is ensured within the interval $[0,1]$, then, for any $k=1,2, \ldots, \# L_{1}(p)$ and $l=1,2, \ldots, \# L_{2}(p)$, the value of $d\left(f\left(L_{1}^{(k)}\right), f\left(L_{2}^{(l)}\right)\right)=$ $\left|f\left(L_{1}^{(k)}\right)-f\left(L_{2}^{(l)}\right)\right|$ must belong to the interval $[0,1]$. According to the expectation of random variable function, one can conclude that the value of $\operatorname{PLWD}_{1}^{h d}\left(L_{1}(p), L_{2}(p)\right)=$ $\inf _{\gamma}\left\{\mathbb{E}_{\gamma} d\left(X_{1}, X_{2}\right)\right\}$ must belong to the interval $[0,1]$.

(2) On the one hand, it is easy to get $\operatorname{PLWD}_{1}^{h d}\left(L_{1}(p), L_{2}(p)\right)=1$ if either $L_{1}(p)=\left\{s_{\tau}(1)\right\}$ and $L_{2}(p)=\left\{s_{-\tau}(1)\right\}$ or $L_{1}(p)=\left\{s_{-\tau}(1)\right\}$ and $L_{2}(p)=\left\{s_{\tau}(1)\right\}$. On the other hand, if the conclusion that either $L_{1}(p)=$ $\left\{s_{\tau}(1)\right\}$ and $L_{2}(p)=\left\{s_{-\tau}(1)\right\}$ or $L_{1}(p)=\left\{s_{-\tau}(1)\right\}$ and $L_{2}(p)=\left\{s_{\tau}(1)\right\}$ is not true, there is at least one $k$ and $l$ satisfying $\left|f\left(L_{1}^{(k)}\right)-f\left(L_{2}^{(l)}\right)\right|<1$, and thus $\operatorname{PLWD}_{1}^{\text {hd }}\left(L_{1}(p), L_{2}(p)\right) \neq 1$.

As far as the distance measure PLWD $_{1}^{\text {hd }}$ is concerned, if the joint probability distribution $(\gamma)$ of two discrete random vectors $X_{1}$ and $X_{2}$ is equipped with the joint probability mass function: $p^{(k l)}=P\left(X_{1}=f\left(L_{1}^{(k)}\right), X_{2}=f\left(L_{2}^{(l)}\right)\right)$, $k=1,2, \ldots, \# L_{1}(p), l=1,2, \ldots, \# L_{2}(p)$, the issue of distance measure by equation (18) can be equivalently transformed into solving an optimization model (M-1) as follows.

$$
\begin{array}{ll}
\min \quad & F\left(p^{(11)}, \ldots, p^{(k l)}, \ldots, p^{\left(\# L_{1}(p) \# L_{2}(p)\right)}\right)=\sum_{k=1}^{\# L_{1}(p)} \sum_{l=1}^{\# L_{2}(p)}\left|f\left(L_{1}^{(k)}\right)-f\left(L_{2}^{(l)}\right)\right| \cdot p^{(k l)} \\
\text { s.t. } \quad\left\{\begin{array}{l}
0 \leq p^{(k l)} \leq 1, \quad k=1,2, \ldots, \# L_{1}(p), l=1,2, \ldots, \# L_{2}(p) \\
\sum_{l=1}^{\# L_{2}(p)} p^{(k l)}=p_{1}^{(k)}, \quad k=1,2, \ldots, \# L_{1}(p) \\
\sum_{k=1}^{\# L_{1}(p)} p^{(k l)}=p_{2}^{(l)}, \quad l=1,2, \ldots, \# L_{2}(p) \\
\sum_{k=1}^{\# L_{1}(p)} p_{1}^{(k)}=\sum_{l=1}^{\# L_{2}(p)} p_{2}^{(l)}=1 .
\end{array}\right.
\end{array}
$$

The objective function in (20) in model (M-1) represents the sum of the weighted distance between two linguistic terms $L_{1}^{(k)}$ in $L_{1}(p)$ and $L_{2}^{(l)}$ in $L_{2}(p)$, whose weights are the joint probability $p^{(k l)}\left(k=1,2, \ldots, \# L_{1}(p) ; l=1,2, \ldots, \# L_{2}(p)\right)$. The first constraint in model (M-1) reflects the basic requirements of the probability mass function. The remaining constraints in Model (M-1) indicate the essential qualification for two marginal distributions $\left(\mu_{1}\right.$ and $\left.\mu_{2}\right)$ of $\gamma$. Therefore, the economic meaning of minimizing the objective function subject to those constraints is to achieve the minimum cost of the weighted distance as the distance measure between two PLTSs. Once model (M-1) is solved, we have found the optimal value, that is, the 1-order PL-Wasserstein distance PLWD ${ }_{1}^{\text {hd }}$ between two given PLTSs $\left(L_{1}(p)\right.$ and $\left.L_{2}(p)\right)$.

Theorem 1. Model (M-1) must have an optimal solution.

Proof. First, the feasible region of model (M-1) is a nonempty set since one can always construct independent random variables with prescribed marginals. Second, the 
feasible region of model (M-1) is bounded under constraints (20). With consideration of the above two aspects, based on the fundamental theorem of linear programming, we can conclude that the optimal value of model (M-1) must be attained.

Computing model (M-1) is based on solving the wellknown transportation problem that is a special type of linear programming, for which the traditional method includes the transportation simplex method and the interior-point method [61]. Therefore, by using Microsoft Excel solver, LINGO, or MATLAB software packages, the transportation problems such as model (M-1) can be solved quickly and effectively.

Example 4. (continued from Examples 1 and 2). In particular, given $d(x, y)=|x-y|$ and $q=1$. By model (M-1), $\operatorname{PLWD}_{1}^{h d}\left(L_{1}(p), L_{2}(p)\right)=(4 / 15), \operatorname{PLWD}_{1}^{h d}\left(L_{1}(p), L_{3}(p)\right)=$ $(1 / 6)$, and $\operatorname{PLWD}_{1}^{h d}\left(L_{2}(p), L_{3}(p)\right)=(1 / 5)$, which is consistent with the results of Example 3. Thus, the proposed PL-Wasserstein distance measure is reasonable and effective.

Remark 5. Compared with the existing PLTSs-based distance measures, the PL-Wasserstein distance measure has the following advantages.

(a) It is not required to add some linguistic terms to PLTSs (such as the distance in Definition 10), which avoids information bias caused by the addition of artificial linguistic terms.

(b) There is no need to rearrange elements of PLTSs by using some ranking rules (such as the distances in Definitions 10 and 11), which eliminates this possibility that different rearrangement rules result in different distance results.

(c) Some basic axioms of a metric or distance (such as identity, symmetry, and triangle inequality) are satisfied, which ensures that the novel measure is a true metric in the mathematical sense.

(d) To adjust the probability set of two PLTSs to be the same (such as the distance in Definitions 10 and 12) is not required, which can greatly improve computational efficiency.

\section{A Novel Adaptive Consensus Reaching Model for Probabilistic Linguistic MAGDM}

To solve the MAGDM problem presented in this paper, our method uses the following five steps: (a) construct an attribute weight determining model (see Section 5.1); (b) establish an expert weight-updating model (see Section 5.2); (c) set up an adaptive assessment-adjusting model (see Section 5.3); (d) develop an adaptive CRP with PLDMs (see Section 5.4); and (e) determine the consensus ranking order of the alternatives (see Section 5.5).

5.1. An Optimization Model for Determining Attribute Weights. In MAGDM, the attribute weights are crucial to the optimal ranking of the alternatives. The weight-determining methods mainly include subjective methods, objective methods, and integrated methods. Among them, the CCSD (correlation coefficient and standard deviation) method has several advantages over other objective methods of weight determination as follows [63]. (a) Compared with the traditional entropy method, it does not require specific normalization methods. (b) It determines more comprehensive and convincing attribute weights than the entropy method and the SD method. (c) It has a clearer modeling mechanism than the CRITIC method. Inspired by the classical CCSD method, we develop an attribute weight determining model, where the new PL-Wasserstein distance measure is combined with the classical CCSD idea.

Firstly, suppose that the assessment value given by expert $e_{k}$ for alternative $a_{i}$ with respect to attribute $c_{j}$ is denoted as $r_{i j}^{(k)}$ in the form of PLTS. To unify the criteria to beneficial type, transform $R^{(k)}=\left(r_{i j}^{(k)}\right)_{m \times n}$ into $\bar{R}^{(k)}=\left(\bar{r}_{i j}^{(k)}\right)_{m \times n}$ by using the following equation:

$$
\bar{r}_{i j}^{(k)}= \begin{cases}r_{i j}^{(k)}, & C_{j} \in C^{\text {benefit }} \\ \left(r_{i j}^{(k)}\right)^{c}, & C_{j} \in C^{\text {cost }}\end{cases}
$$

where the supplement operation of PLTS $r_{i j}^{(k)}$ is expressed as [64]

$$
\left(r_{i j}^{(k)}\right)^{c}=f^{-1}\left(\bigcup_{\delta_{i j}^{(l(k))} \in f\left(L_{i j}^{(k)}(p)\right)}\left\{\left(1-\delta_{i j}^{(l(k))}\right)\left(p_{i j}^{(l(k))}\right)\right\}\right), \quad l(k)=1,2, \ldots, \# L_{i j}^{(k)}(p) .
$$

Subsequently, utilize Definition 4 to transform the $\bar{R}^{(k)}=$ $\left(\bar{r}_{i j}^{(k)}\right)_{m \times n}$ to the normalized individual PLDMs $\widehat{R}^{(k)}=$ $\left(\widehat{r}_{i j}^{(k)}\right)_{m \times n}, k=1,2, \ldots, h$.

Then, based on the above $h$ normalized individual PLDMs, an incomplete attribute weight information set $\Omega$, and an expert weight vector $\lambda=\left(\lambda^{(1)}, \lambda^{(2)}, \ldots, \lambda^{(h)}\right)^{T}$, the specific procedure of determining attribute weights is shown as follows.

On the one hand, by utilizing Definition 7 , we can obtain the positive-ideal and negative-ideal solution of attributes $c_{j}$ associated with expert $e_{k}$, which are expressed as $\hat{r}_{j}^{+(k)}=$ $\max \left(\widehat{r}_{1 j}^{(k)}, \widehat{r}_{2 j}^{(k)}, \ldots, \widehat{r}_{m j}^{(k)}\right)$ and $\widehat{r}_{j}^{-(k)}=\min \left(\widehat{r}_{1 j}^{(k)}, \widehat{r}_{2 j}^{(k)}, \ldots, \widehat{r}_{m j}^{(k)}\right)$, respectively. Based on the proposed PL-Wasserstein distance measure, we construct a normalized numerical performance matrix $D^{(k)}=\left(d_{i j}^{(k)}\right)_{m \times n}$, whose entries are represented by

$$
d_{i j}^{(k)}=\frac{\operatorname{PLWD}_{1}^{h d}\left(\widehat{r}_{i j}^{(k)}, \widehat{r}_{j}^{-(k)}\right)}{\operatorname{PLWD}_{1}^{h d}\left(\widehat{r}_{j}^{+(k)}, \widehat{r}_{j}^{-(k)}\right)}
$$


Given the simple additive weighting (SAW) method, the numerical performance value of each alternative associated with expert $e_{k}$ can be calculated through the expression $v_{i}^{(k)}=\sum_{j=1}^{n} w^{(j)} d_{i j}^{(k)}, j=1,2, \ldots, n$. Generally, as far as the expert $e_{k}$ is concerned, the greater performance value $v_{i}^{(k)}$ for alternative $a_{i}$, the better the alternative $a_{i}$. The best alternative (option) is the option that has the highest performance value. Now, to take into account the impact of the attribute $c_{j}$ on decision making, we remove it from the set of attributes. When $c_{j}$ is eliminated, the overall performance value of alternative $a_{i}$ is determined by

$$
g_{i j}^{(k)}=\sum_{t=1, t \neq j}^{n} w^{(t)} d_{i t}^{(k)} .
$$

Then, the correlation coefficient $\rho_{j}^{(k)}$ between the performance value of $c_{j}$ with respect to expert $e_{k}$ and the above overall performance value is defined as

$$
\rho_{j}^{(k)}=\frac{\sum_{i=1}^{m}\left[\left(d_{i j}^{(k)}-(1 / m) \sum_{i=1}^{m} d_{i j}^{(k)}\right) \times\left(g_{i j}^{(k)}-(1 / m) \sum_{i=1}^{m} g_{i j}^{(k)}\right)\right]}{\sqrt{\sum_{i=1}^{m}\left(d_{i j}^{(k)}-(1 / m) \sum_{i=1}^{m} d_{i j}^{(k)}\right)^{2}} \times \sqrt{\sum_{i=1}^{m}\left(g_{i j}^{(k)}-(1 / m) \sum_{i=1}^{m} g_{i j}^{(k)}\right)^{2}}} .
$$

If $\rho_{j}^{(k)}$ is large enough to be close to 1 , then the rating value of attribute $c_{j}$ and the total performance value excluding attribute $c_{j}$ will have nearly the same numerical distributions and rankings. In this case, the removal of the attribute $c_{j}$ will have little impact on decision making. Therefore, the attribute $c_{j}$ should be assigned a low weight. If $\rho_{j}^{(k)}$ is small enough to be close to -1 , then the assessment value of attribute $c_{j}$ and the total performance value without the inclusion of $c_{j}$ will have almost opposite numerical distributions and rankings. In this case, the removal of the attribute $c_{j}$ will have a great impact on decision making. So, the attribute $c_{j}$ should be assigned a high weight.

On the other hand, the larger the standard deviation of an attribute, the greater the variation degree of the value of the attribute, indicating that the attribute with larger standard deviation has a greater impact on the decision making. So, the attributes with the larger standard deviation should have greater weights than those with lower standard deviation. The standard deviation of attribute $c_{j}$ with regard to expert $e_{k}$ is formulated as

$$
\sigma_{j}^{(k)}=\sqrt{\frac{1}{m-1} \sum_{i=1}^{m}\left(d_{i j}^{(k)}-\frac{1}{m} \sum_{i=1}^{m} d_{i j}^{(k)}\right)^{2}} .
$$

Based on the consideration of the above two aspects, when giving the expert weight vector $\lambda=\left(\lambda^{(1)}, \lambda^{(2)}, \ldots, \lambda^{(h)}\right)^{T}$ in advance, we can construct the following nonlinear optimization model (M-2) for objectively determining attribute weights involved in MAGDM problems investigated in this paper.

$$
\begin{aligned}
& \min J(w)=\sum_{k=1}^{h} \lambda^{(k)} \sum_{j=1}^{n}\left(w^{(j)}-\frac{\sigma_{j}^{(k)} \sqrt{1-\rho_{j}^{(k)}}}{\sum_{j=1}^{n} \sigma_{j}^{(k)} \sqrt{1-\rho_{j}^{(k)}}}\right)^{2} \\
& \text { s.t. }\left\{\begin{array}{l}
w=\left(w^{(1)}, w^{(2)}, \ldots, w^{(n)}\right) \in \Omega \\
\sum_{j=1}^{n} w^{(j)}=1, \quad w^{(j)} \geq 0, j=1,2, \ldots, n .
\end{array}\right.
\end{aligned}
$$

In model (M-2), the objective function $J(w)$ indicates the total error of estimating the attribute weights for all experts with the consideration of two factors involved the correlation coefficient and the standard deviation, where the root value of $1-\rho_{j}^{(k)}$ is used to reduce the difference between the largest and the smallest weights that are directly estimated through the correlation coefficient $\rho_{j}^{(k)}$. The first constraint in model (M-2) reflects the set of incomplete weight information that is compatible with experts' subjective preferences. The second constraint in model (M-2) embodies the non-negative and normalization requirements of attribute weights. Using MATLAB and LINGO software packages, we can solve this nonlinear model to obtain the weight of each attribute.

5.2. Expert Weight Updating Model That Considers the Dynamic Individual Cumulative Contribution. Consensus measure is an important step to measure the consensus level among experts in MAGDM problems. Taking into consideration the characteristics of the MAGDM problem in which experts' assessments are characterized by PLTSs, we need to reconstruct an effective method for measuring the individual and group consensus. Inspired by the existing 
consensus improving strategies $[45,46]$, a new approach to measuring experts' consensus contribution to the group and the group consensus level is given below.

First, using equation (11), the collective assessment of all experts for alternative $a_{i}$ with respect to attribute $c_{j}$ can be formulated as

$$
\widehat{r}_{i j}^{(G)}=\sum_{k=1}^{h} \widehat{r}_{i j}^{(k)} \cdot \lambda^{(k)}
$$

So, we can obtain the collective PLDM $\widehat{R}^{(G)}=\left(\widehat{r}_{i j}^{(G)}\right)_{m \times n}$.

Then, the following individual consensus degree of expert $e_{l}$ on alternative $a_{i}$ can be expressed by

$$
\operatorname{ICL}_{i}^{(l)}=1-\sum_{j=1}^{n} w^{(j)} \cdot \operatorname{PLWD}_{1}^{h d}\left(\widehat{r}_{i j}^{(l)}, \widehat{r}_{i j}^{(G)}\right),
$$

where PLWD ${ }_{1}$ means 1-Wasserstein distance that can be computed by model (M-1) and $w^{(j)}$ that can be calculated by model (M-2) represents the weight of attribute $c_{j}$.

Subsequently, the consensus degree of all experts on alternative $a_{i}$ can be obtained by

$$
\mathrm{CL}_{i}=\sum_{k=1}^{h} \lambda^{(k)} \cdot \operatorname{ICL}_{i}^{(k)}=\sum_{k=1}^{h}\left(1-\sum_{j=1}^{n} w^{(j)} \cdot \operatorname{PLWD}_{1}^{h d}\left(\widehat{r}_{i j}^{(k)}, \widehat{r}_{i j}^{(G)}\right)\right) \cdot \lambda^{(k)} .
$$

Similar to equation (30), the consensus degree $\mathrm{CL}_{i}^{(l)}$ on alternative $a_{i}$ without expert $e_{l}$ can be obtained by the following equation:

$$
\mathrm{CL}_{i}^{(l)}=\sum_{k \in E_{s} \backslash\{l\}}\left(1-\sum_{j=1}^{n} w^{(j)} \cdot \operatorname{PLWD}_{1}^{h d}\left(\widehat{r}_{i j}^{(k)}, \widehat{r}_{i j}^{(G)}\right)\right) \cdot \beta^{(k)} .
$$

In equation (31), $\beta^{(k)}=\left(\lambda^{(k)} / \sum_{k \in E_{s} \backslash\{l\}} \lambda^{(k)}\right), i=1, \ldots, m$, $l=1, \ldots, h$, and $k \in E_{s} \backslash\{l\}$ indicates that $k \in E_{s}, k \neq l$ where $E_{s}$ represents the subscript set of all elements belonging to the expert set $E$.

Definition 14. Suppose $\mathrm{CL}_{i} \quad(i=1, \ldots, m)$ and $\mathrm{CL}_{i}^{(l)}$ $(i=1, \ldots, m, l=1, \ldots, h)$ have the meanings as shown in equations (30) and (31), respectively. Then, for the alternative set $A$ with respect to the attribute set $C$, the ICCC degree of expert $e_{l}(l=1, \ldots, h)$ can be defined as

$$
\mathrm{ICD}^{(l)}=\sum_{i=1}^{m}\left(\mathrm{CL}_{i}-\mathrm{CL}_{i}^{(l)}\right) \text {. }
$$

Here, $\mathrm{ICD}^{(l)}$ reflects the individual contribution degree of expert $e_{l}$ who devotes oneself to the CRPs. If $\operatorname{ICD}^{(l)}>0$, it shows that the expert $e_{l}$ plays a positive role in CRPs. The larger value of $\mathrm{ICD}^{(l)}$ indicates the higher consensus contribution that expert $e_{l}$ makes to the group decision. On the contrary, if $\operatorname{ICD}^{(l)}<0$, it indicates that the opinion of expert $e_{l}$ has a negative influence on the CRPs.

Definition 15. The group consensus degree on all the alternatives can be defined as

$$
\begin{aligned}
\mathrm{GCD}^{(G)} & =\frac{1}{m} \sum_{i=1}^{m} \mathrm{CL}_{i} \\
& =\frac{1}{m} \sum_{i=1}^{m} \sum_{k=1}^{h}\left(1-\sum_{j=1}^{n} w^{(j)} \cdot \operatorname{PLWD}_{1}^{h d}\left(\widehat{r}_{i j}^{(k)}, \widehat{r}_{i j}^{(G)}\right)\right) \cdot \lambda^{(k)},
\end{aligned}
$$

where $\operatorname{GCD}^{(G)}(\in \in[0,1])$ reflects the consensus level of the expert group. The larger the value of $\mathrm{GCD}^{(G)}$, the higher the consensus level of the group.

In general, the group consensus threshold $\eta$ needs to be determined in advance, and its determination methods mainly include the subjective experience method and objective simulation analysis method $[42,65]$. If the group consensus level $\mathrm{GCD}^{(G)}$ does not meet the requirement of consensus threshold $\eta$ (that is, $\mathrm{GCD}^{(G)}<\eta$ ), the system may reevaluate the individual cumulative consensus contribution $\operatorname{ICD}^{(l)}$, and then adaptively update the weight of expert $e_{l}$ according to the value of $\mathrm{ICD}^{(l)}$ so as to improve the consensus level of the group in the next round of consensus iteration. The ICCC-based updating method of expert weights can be described as follows.

Suppose that the weight and the ICCC degree of expert $e_{l}$ are denoted as $\lambda^{(l, t)}$ and $\operatorname{ICD}^{(l, t)}$ in the $t$-th iteration, respectively. Then, the weight of expert $e_{l}$ in the $(t+1)$-th iteration can be adaptively updated by applying equations (34) and (35).

$$
\begin{aligned}
\lambda^{(l, t+1)} & =\frac{\mu^{(l, t+1)}}{\sum_{l=1}^{h} \mu^{(l, t+1)}}, \\
\mu^{(l, t+1)} & =\lambda^{(l, t)} \cdot\left(1+\operatorname{ICD}^{(l, t)}\right)^{\theta} .
\end{aligned}
$$

Here, the parameter $\theta$ represents the influence of the contribution of the expert on his/her weight. The higher the value of the parameter $\theta$, the faster the process converges to the desired consensus level. A detailed discussion of the parameter $\theta$ can be found in the literature [66]. So, the meaning of integrating equations (34) and (35) is to directly give greater weights to the experts with more ICCC and to punish the weights of the experts with less ICCC. That is, experts who contribute more to the group decision improve their importance while experts who are contrary to the group lose some of their weights.

5.3. Adaptive Assessment-Adjusting Model Composed of Identification and Modification Process. The assessmentadjusting process consists of two parts: (a) identification and (b) modification. In the process of identification, the experts whose evaluations are not consistent with the decision group will be identified. The identification process consists of the following three steps.

First, the set of experts that should change their opinions, which is called EXC, are those who have the least contributions to reaching consensus.

$$
\mathrm{EXC}=\left\{e_{k^{*}} \mid \mathrm{ICD}^{\left(k^{*}\right)}=\min _{1 \leq k \leq h} \operatorname{ICD}^{(k)}\right\} .
$$


Then, for any expert $e_{k^{*}} \in \mathrm{EXC}$, the alternatives related to this set have the least individual consensus degree, which is denoted as ALT as follows.

$$
\operatorname{ALT}=\left\{a_{i^{*}} \mid\left(e_{k^{*}} \in \operatorname{EXC}\right) \wedge\left(\operatorname{ICL}_{i^{*}}^{\left(k^{*}\right)}=\min _{1 \leq i \leq m} \operatorname{ICL}_{i}^{\left(k^{*}\right)}\right)\right\} .
$$

Finally, for any expert $e_{k^{*}} \in \mathrm{EXC}$ and $a_{i^{*}} \in \mathrm{ALT}$, the following rule is used to identify the individual assessment value $\widehat{r}_{i^{*} j^{*}} k^{*}$ with a maximum distance from the group opinion. These values to be modified are denoted as EVM which is selected as

$$
\operatorname{EVM}=\left\{\widehat{r}_{i^{*} j^{*}}^{\left(k^{*}\right)} \mid\left(e_{k^{*}} \in \operatorname{EXC}\right) \wedge\left(a_{i^{*}} \in \operatorname{ALT}\right) \wedge\left(\operatorname{PLWD}_{1}^{h d}\left(\widehat{r}_{i^{*} j^{*}}^{\left(k^{*}\right)}, \widehat{r}_{i^{*} j^{*}}^{(G)}\right)=\max _{1 \leq j \leq n} \operatorname{PLWD}_{1}^{h d}\left(\widehat{r}_{i^{*} j}^{\left(k^{*}\right)}, \widehat{r}_{i^{*} j}^{(G)}\right)\right)\right\}
$$

In the process of modification, the identified experts need to adaptively modify their assessment information. Suppose that the individual assessment of expert $e_{k}$ on alternative $a_{i}$ with respect to attribute $c_{j}$ and the collective assessment are denoted as $\widehat{r}_{i j}^{(k, t)}$ and $\widehat{r}_{i j}^{(G, t)}$ in the $t$-th iteration, respectively. After using equations (36)-(38), to identify the position $\left(k^{*}, i^{*}, j^{*}\right)$ of individual assessment value $\widehat{r}_{i^{*} j^{*}}^{\left(k^{*}\right)}$ that needs to be modified, apply equation (39) to adaptively adjust the corresponding assessment value in the $(t+1)$-th iteration.

$$
\widehat{r}_{i j}^{(k, t+1)}= \begin{cases}\xi \cdot \widehat{r}_{i j}^{(k, t)} \oplus(1-\xi) \cdot \widehat{r}_{i j}^{(G, t)}, & \text { if } k=k^{*}, i=i^{*}, j=j^{*} \\ \widehat{r}_{i j}^{(k, t)}, & \text { otherwise, }\end{cases}
$$

where $\widehat{r}_{i j}^{(G, t)}=\sum_{k=1}^{h} \widehat{r}_{i j}^{(k)} \cdot \lambda^{(k, t)}$ by using equation (28), “ $\oplus$ " indicates the addition operation of PLTSs [64], and the parameter $\xi$ denotes the adjustment coefficient.

5.4. The Proposed Adaptive CRP. In what follows, to obtain the optimal alternative accepted by the group of DMs, we put forward a novel adaptive CRP for probabilistic linguistic MAGDM. In the consensus process, we propose to combine the weight-updating model and assessment-adjusting model (called an integrated consensus improving strategy) to improve a high consensus level that is measured by applying PL-Wasserstein distance measure. If the consensus degree satisfies the condition $\mathrm{GCD}^{(G)}>\eta$, the decision-making process ends and the best alternative is obtained. Otherwise, the experts' weights and assessments need to be adjusted according to the integrated consensus improving technique. The flowchart of the adaptive CRP is shown in Figure 3.

The detailed algorithm of the adaptive CRP, called Algorithm 1, is described as follows.

\subsection{Probabilistic Linguistic MAGDM Approach with the Novel} Proposed CRP. Following the above analysis, to ensure that the group can reach a consensus in terms of the ranking of alternatives and the choice of the best alternative(s), a probabilistic linguistic MAGDM approach with the aforementioned adaptive CRP, called Algorithm 2, is proposed. We can now describe the main steps of the proposed MAGDM approach.
In the proposed MAGDM approach (Algorithm 2), Steps 1 and 2 indicate the preparation phase of consensus-driven probabilistic linguistic MAGDM discussed in this paper. Step 3 describes a new CRP, which is called an adaptive CRP with PLTSs based on PL-Wasserstein distance and an integrated consensus improving technique. The selection process is reflected in Steps 4 and 5.

\section{Case Study}

In this section, the proposed consensus-driven MAGDM method is applied to the evaluation of real network public opinion emergencies.

6.1. Case Background. Recently, three Internet public opinion emergencies $A=\left\{a_{1}, a_{2}, a_{3}\right\}$ have recently broken out in a city. Under the condition of limited rescue resources, the relevant departments must determine the priority of dealing with Internet public opinion emergencies based on the comprehensive severity of each emergency. To do this, they firstly select three different types of decision experts representing different emergency interests to form an emergency decision-making committee $E=\left\{e_{1}, e_{2}, e_{3}\right\}$ (i.e., government departments $\left(e_{1}\right)$, online media $\left(e_{2}\right)$, and netizens $\left.\left(e_{3}\right)\right)$. The crisis assessment of network public opinion emergencies involves emergency management and information dissemination. Information amount, diffusion degree, audience tendency, and response status of online public opinion mainly affect the judgment and decision of emergency experts on the degree of comprehensive severity of each network public opinion emergency [4]. For this reason, the emergency management experts select four significant assessment criteria of public opinion crisis (i.e., the content sensitivity and form richness of Internet public opinion ( $c_{1}$, benefit); the influence and transmission of media ( $c_{2}$, benefit); the participation, attitudes and the emotional tendency of netizens ( $c_{3}$, benefit); and the emergency capability of governments $\left(c_{4}\right.$, cost $\left.)\right)$. Each emergency expert separately evaluates the above three network public opinion emergencies under the above four criteria. The LTSs used on the first three criteria are $S_{1}=\left\{s_{-3}=\right.$ very low,$s_{-2}=$ low,$s_{-1}=$ slightly low,$s_{0}=$ medium, $s_{1}=$ slightly high, $s_{2}=$ high,$s_{3}=$ very high $\}$, and LTS for the fourth criterion is $S_{2}==\left\{s_{-3}=\right.$ very weak, $s_{-2}=$ weak, 
$s_{-1}=$ slightly weak,$s_{0}=$ medium, $s_{1}=$ slightly strong, $s_{2}=$ strong, $s_{3}=$ very strong $\}$. The decision maker recorded the linguistic evaluation information of the experts on each emergency under each indicator. The collected linguistic assessment values that are provided by all the experts with the same interest group are, respectively, stored in the form of a data table expressed by a PLDM, where each data unit is a PLTS. Furthermore, the data unit can also imply the missing partial information of some experts, that is, the sum of probabilities of linguistic terms in each PLTS can be less than 1. The original PLDMs, provided by three different types of decision experts, are shown in Tables 1-3, respectively. The problem we need to solve is how to obtain the consensus ranking of the above Internet public opinion crisis rating and thus to select the most severe one that needs to be prioritized under the premise of ensuring the consensus reaching within the group of experts.

6.2. Decision-Making Process. The method proposed in this paper used the following steps.

Step 1: as Step 1 is given above, we start our computation from Step 2.

Step 2: by Definition 4 and equations (21) and (22), we obtain the following normalized individual PLDMs $\widehat{R}^{(k)}=\left(\widehat{r}_{i j}^{(k)}\right)_{3 \times 4}(k=1,2,3)$.

$$
\begin{aligned}
& \widehat{R}^{(1)}=\left(\begin{array}{cccc}
\left\{s_{-1}(0.4), s_{0}(0.6)\right\} & \left\{s_{1}(0.8), s_{2}(0.2)\right\} & \left\{s_{1}(1)\right\} & \left\{s_{0}(0.6), s_{1}(0.4)\right\} \\
\left\{s_{2}(1)\right\} & \left\{s_{0}(0.6), s_{1}(0.4)\right\} & \left\{s_{2}(0.7), s_{3}(0.3)\right\} & \left\{s_{-1}(0.5), s_{0}(0.5)\right\} \\
\left\{s_{0}(0.5), s_{1}(0.5)\right\} & \left\{s_{2}(1)\right\} & \left\{s_{0}(0.6), s_{1}(0.4)\right\} & \left\{s_{-2}(0.2), s_{-1}(0.8)\right\}
\end{array}\right), \\
& \widehat{R}^{(2)}=\left(\begin{array}{cccc}
\left\{s_{-1}(1)\right\} & \left\{s_{2}(1)\right\} & \left\{s_{0}(0.5), s_{1}(0.5)\right\} & \left\{s_{1}(1)\right\} \\
\left\{s_{2}(0.5), s_{3}(0.5)\right\} & \left\{s_{0}(0.5), s_{1}(0.5)\right\} & \left\{s_{2}(1)\right\} & \left\{s_{0}(1)\right\} \\
\left\{s_{0}(1)\right\} & \left\{s_{2}(0.6), s_{3}(0.4)\right\} & \left\{s_{-1}(0.2), s_{0}(0.8)\right\} & \left\{s_{-1}(1)\right\}
\end{array}\right), \\
& \widehat{R}^{(3)}=\left(\begin{array}{cccc}
\left\{s_{1}(1)\right\} & \left\{s_{1}(0.5), s_{2}(0.5)\right\} & \left\{s_{0}(1)\right\} & \left\{s_{-1}(1)\right\} \\
\left\{s_{0}(1)\right\} & \left\{s_{1}(0.6), s_{2}(0.4)\right\} & \left\{s_{2}(1)\right\} & \left\{s_{-1}(0.8), s_{0}(0.2)\right\} \\
\left\{s_{-1}(1)\right\} & \left\{s_{2}(1)\right\} & \left\{s_{-1}(1)\right\} & \left\{s_{-2}(1)\right\}
\end{array}\right) .
\end{aligned}
$$

Step 3: we select the linguistic scale function shown as equation (4) for criteria $c_{1}$ and $c_{3}$ and the linguistic scale function shown as equation (5) for criteria $c_{2}$ and $c_{4}$ with the two parameters $\gamma=\zeta=1.5$. Suppose that the initial weight of each expert is the same (i.e., $\left.\lambda^{(1)}=\lambda^{(2)}=\lambda^{(3)}=(1 / 3)\right)$. The incomplete attribute weight information set $\Omega=R^{4}$, the maximum number of iterations $t^{\max }=30$, the group consensus threshold $\eta=0.95$, and the two parameters $\theta=1.5, \xi=0.1$.
Subsequently, we conduct the proposed adaptive CRP by Algorithm 1. The main procedure of the adaptive CRP is as follows.

Substep 1: let $t=0$; we have $\widehat{R}^{(k, 0)}=\widehat{R}^{(k)}, \lambda^{(k, 0)}=\lambda^{(k)}$, $k=1,2,3$.

Substep 2: using equation (28), we obtain the temporary collective PLDM.

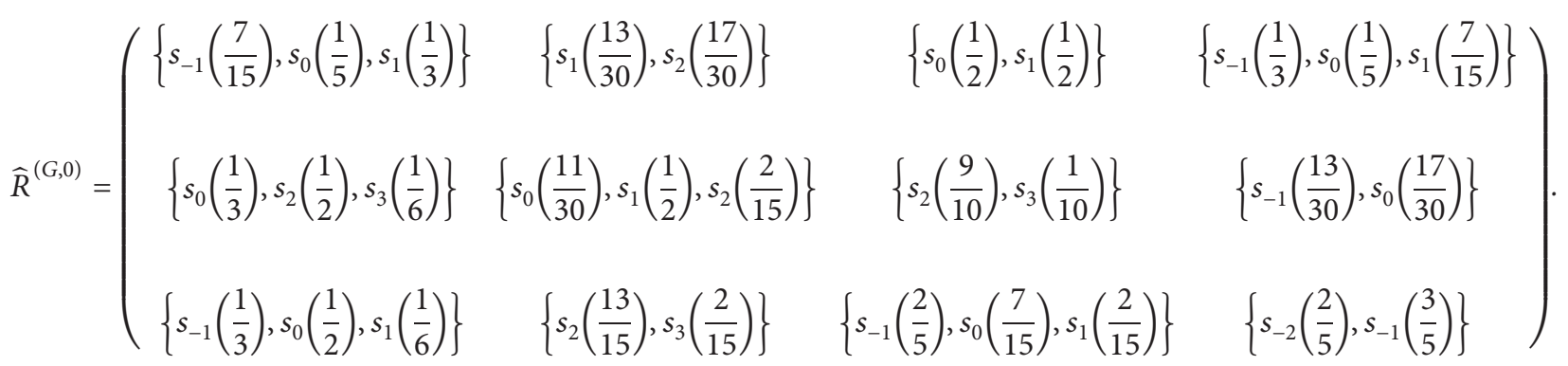




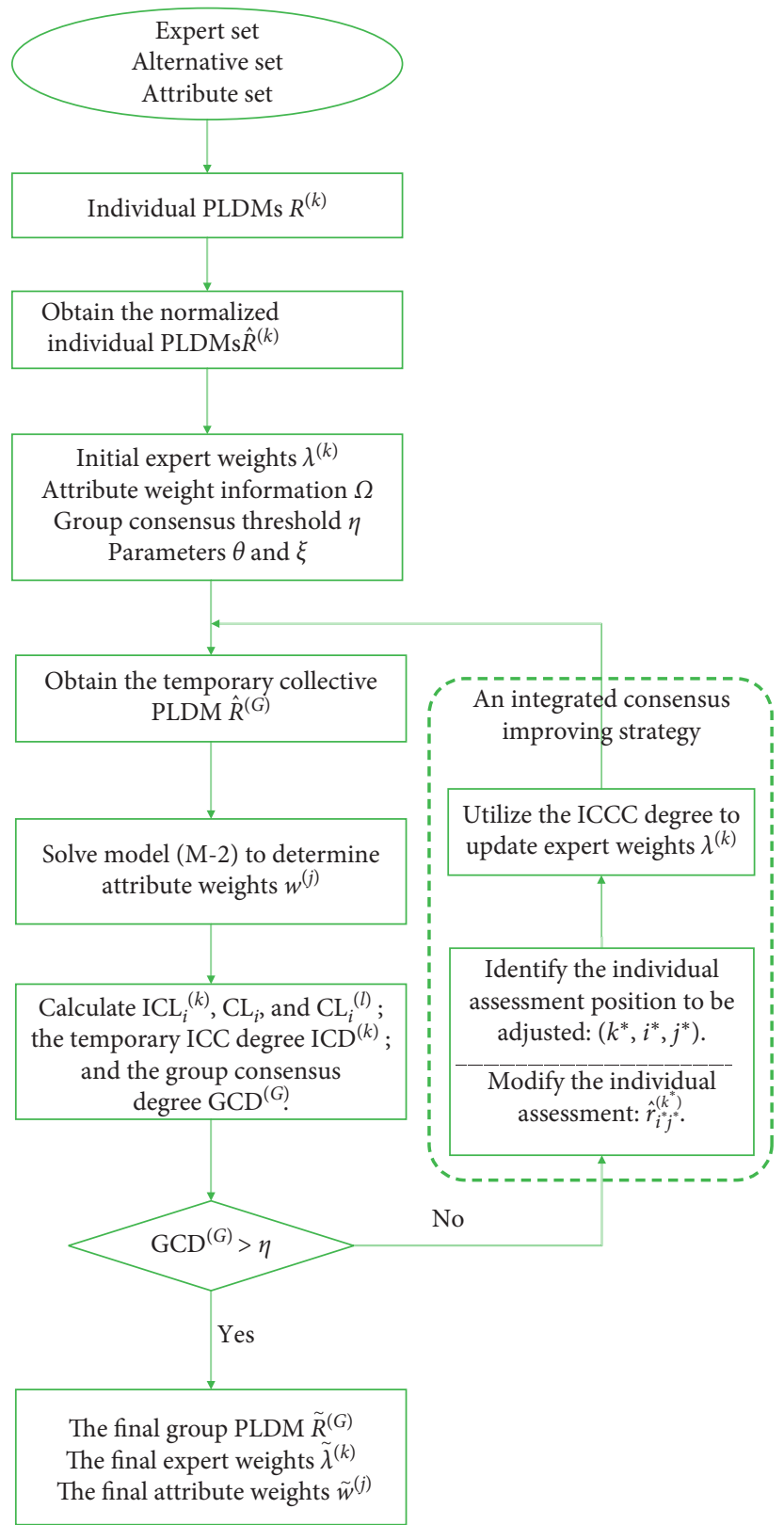

FIgURE 3: The flowchart of the proposed adaptive CRP.

Substep 3: solving model (M-2), we obtain the following attribute weights:

$$
\begin{aligned}
& w^{(1,0)}=0.2108, \\
& w^{(2,0)}=0.2533, \\
& w^{(3,0)}=0.3206, \\
& w^{(4,0)}=0.2153 .
\end{aligned}
$$

Substep 4: by equations (29)-(31), we calculate $\mathrm{ICL}_{i}^{(l)}$, $\mathrm{CL}_{i}$, and $\mathrm{CL}_{i}^{(l)}, i=1,2,3, l=1,2,3$. Detailed results are shown in Tables 4-6.

Using equation (32), we calculate the temporary ICCC degree: $\quad \operatorname{ICD}^{(1,0)}=0.0146, \quad \operatorname{ICD}^{(2,0)}=0.0145$, and
$\operatorname{ICD}^{(3,0)}=-0.0290$. Using equation (33), we get the temporary group consensus degree $\mathrm{GCD}^{(G, 0)}=0.9265$. Since $\operatorname{GCD}^{(G, 0)}<\eta=0.95$ and $t<t^{\max }$, then go to Substep 5.

Substep 5: based on equations (36)-(38), we have $\mathrm{EXC}=\left\{e_{3}\right\}, \operatorname{ALT}=\left\{a_{3}\right\}$, and $\mathrm{EVM}=\left\{\widehat{r}_{31}^{(3,0)}\right\}$. So, we identify the individual assessment $\widehat{r}_{31}^{(3,0)}$ that needs to be adjusted, namely, the assessment of expert $e_{3}$ on emergency $a_{3}$ with respect to attribute $c_{1}$. Meanwhile, by equation (39) and the addition operation of PLTSs “ $\oplus$ " [64], we adjust the assessment value as follows:

$$
\begin{aligned}
\hat{r}_{31}^{(3,1)} & =0.1 \cdot \widehat{r}_{31}^{(3,0)} \oplus 0.9 \cdot \widehat{r}_{31}^{(G, 0)} \\
& =\left\{s_{-1}(0.3333), s_{-0.0876}(0.5), s_{0.8565}(0.1667)\right\} .
\end{aligned}
$$


Input: The normalized individual PLDMs $\widehat{R}^{(k)}=\left(\widehat{r}_{i j}^{(k)}\right)_{m \times n}$ and their associated initial weights $\lambda^{(k)}(k=1,2, \ldots, h)$, the incomplete attribute weight information set $\Omega$, the maximum number of iterations $t^{\max } \geq 1$, the group consensus threshold $\eta(0<\eta<1)$, and the parameters $\theta$ and $\xi$.

Output: The terminal iteration step $t$, the adjusted individual PLDMs $\widetilde{R}^{(k)}(k=1,2, \ldots, h)$ and their adjusted expert weights $\tilde{\lambda}^{(k)}$ $(k=1,2, \ldots, h)$, the collective PLDM $\widetilde{R}^{(G)}$, the ICCC degree $\widetilde{\mathrm{ICD}}^{(k)}(k=1,2, \ldots, h)$, and the group consensus degree $\widetilde{\mathrm{GCD}}^{(G)}$.

Step 1: Let $t=0$ and $\widehat{R}^{(k, 0)}=\left(\widehat{r}_{i j}^{(k, 0)}\right)_{m \times n}=\left(\widehat{r}_{i j}^{(k)}\right)_{m \times n}=\widehat{R}^{(k)}, \lambda^{(k, 0)}=\lambda^{(k)}, k=1,2, \ldots, h$.

Step 2: Obtain the temporary collective PLDM $\widehat{R}^{(G, t)}=\left(\widehat{r}_{i j}^{(G, t)}\right)_{m \times n}$ using equation (28).

Step 3: Determine the attribute weights $w^{(j, t)}=w^{(j)}(j=1,2, \ldots, n)$ using model (M-2).

Step 4: Calculate $\mathrm{ICL}_{i}^{(k)}, \mathrm{CL}_{i}$, and $\mathrm{CL}_{i}^{(l)}$ by equations (29)-(31). Determine the temporary ICCC degree $\mathrm{ICD}^{(k, t)}$ and the temporary group consensus degree $\mathrm{GCD}^{(G, t)}$ using equations (32) and (33), respectively. Based on the given group consensus threshold $\eta$, we judge whether the consensus degree of the group is acceptable or not. If $\mathrm{GCD}^{(G, t)}<\eta$ and $t<t^{\max }$, then go to the next step; otherwise, go to Step 7.

Step 5: Identify the position $\left(k^{*}, i^{*}, j^{*}\right)$ of individual assessment $\widehat{r}_{i^{*} j^{*}}^{\left(k^{*}\right)}$ contributing less to reaching consensus based on equations (36)-(38) and apply equation (39) to adjust the corresponding assessment values, and then go to Step 6.

Step 6: Update experts' weights $\lambda^{(k, t)}(k=1,2, \ldots, h)$ using equations (34) and (35). Let $t:=t+1$ and go to Step 2.

Step 7: Let $\widetilde{w}^{(j)}=w^{(j, t)}(j=1,2, \ldots, n), \widetilde{R}^{(G)}=\widehat{R}^{(G, t)}$, and $\widetilde{\mathrm{GCD}}^{(G)}=\mathrm{GCD}^{(G, t)}$. Also, let $\widetilde{\lambda}^{(k)}=\lambda^{(k, t)}, \widetilde{R}^{(k)}=\widehat{R}^{(k, t)}$,

$\widetilde{\mathrm{ICD}}^{(k)}=\mathrm{ICD}^{(k, t)}, k=1,2, \ldots, h$. Output the terminal iteration step $t$, the modified individual PLDMs $\widetilde{R}^{(k)}(k=1,2, \ldots, h)$ and their associated weights $\widetilde{\lambda}^{(k)}(k=1,2, \ldots, h)$, the final attribute weights $\widetilde{w}^{(j)}(j=1,2, \ldots, n)$, the final group PLDM $\widetilde{R}^{(G)}$, the ICCC degree $\widetilde{\mathrm{ICD}}^{(k)}(k=1,2, \ldots, h)$, and the group consensus degree $\widetilde{\mathrm{GCD}}^{(G)}$.

Step 8: End.

Algorithm 1: The proposed adaptive CRP.

Step 1: identify all the alternatives to be evaluated, the assessment attributes, and the invited experts. Determine the preestablished LTS $S=\left\{s_{\alpha} \mid \alpha=-\tau, \ldots,-1,0,1, \ldots, \tau\right\}$ and the corresponding linguistic scale functions $f$. Collect the linguistic evaluations from experts and then construct the individual PLDMs $R^{(k)}=\left(r_{i j}^{(k)}\right)_{m \times n}=\left(L_{i j}^{(k)}(p)\right)_{m \times n}(k=1,2, \ldots, h)$, where $L_{i j}^{(k)}(p)$ is in the form of a PLTS.

Step 2: utilize Definition 4 and equations (21) and (22) to obtain the normalized individual PLDMs $\widehat{R}^{(k)}=\left(\widehat{r}_{i j}^{(k)}\right)_{m \times n}(k=1,2, \ldots, h)$.

Step 3: use Algorithm 1 to implement the CRP. If the CRP ends, then go to Step 4.

Step 4: based on the final attribute weight $\widetilde{w}=\left(\widetilde{w}^{(1)}, \widetilde{w}^{(2)}, \ldots, \widetilde{w}^{(n)}\right)^{T}$ and the final group PLDM $\widetilde{R}^{(G)}=\left(\widetilde{r}_{i j}^{(G)}\right)_{m \times n}$, utilize equations (8) and (9) to calculate the general expected value $E\left(\widetilde{r}_{i j}^{(G)}\right)$ and the general variance value $\sigma\left(\widetilde{r}_{i j}^{(G)}\right)$ of the collective assessment value $\widetilde{r}_{i j}^{(G)}$. Then, the weighted arithmetic operator is employed to obtain the weighted general expected value $E\left(\widetilde{R}_{i}^{(G)}\right)$ and the weighted general variance value $\sigma\left(\widetilde{R}_{i}^{(G)}\right)$ of alternative $a_{i}(i=1,2, \ldots, m)$, respectively. $E\left(\widetilde{R}_{i}^{(G)}\right)=\sum_{j=1}^{n} \widetilde{w}^{(j)} \cdot E\left(\widetilde{r}_{i j}^{(G)}\right)$, $\sigma\left(\widetilde{R}_{i}^{(G)}\right)=\sum_{j=1}^{n} \widetilde{w}^{(j)} \cdot \sigma\left(\widetilde{R}_{i j}^{(G)}\right)$.

Step 5: based on the idea of the coefficient of variation [67], construct the measure index $\mathrm{CV}_{i}$ to cope with the performance of alternative $a_{i}$, where $\mathrm{CV}_{i}=\left(E\left(\widetilde{R}_{i}^{(G)}\right) /\left(E\left(\widetilde{R}_{i}^{(G)}\right)+\sigma\left(\widetilde{R}_{i}^{(G)}\right)\right)\right)$.

Generate the ranking order of alternatives according to the increasing values, $\mathrm{CV}_{i}, i=1,2, \ldots, m$. Select the best alternative from the set $A=\left\{a_{1}, a_{2}, \ldots, a_{m}\right\}$.

Step 6: end.

Algorithm 2: The proposed MAGDM approach.

TABLE 1: The PLDM $R^{(1)}$ provided by the government departments $e_{1}$.

\begin{tabular}{ccccc}
\hline & $c_{1}$ & $c_{2}$ & $c_{3}$ & $c_{4}$ \\
\hline$a_{1}$ & $\left\{s_{-1}(0.4), s_{0}(0.6)\right\}$ & $\left\{s_{1}(0.8), s_{2}(0.2)\right\}$ & $\left\{s_{1}(1)\right\}$ & $\left\{s_{-1}(0.4), s_{0}(0.6)\right\}$ \\
$a_{2}$ & $\left\{s_{2}(1)\right\}$ & $\left\{s_{0}(0.6), s_{1}(0.4)\right\}$ & $\left\{s_{2}(0.7), s_{3}(0.3)\right\}$ & $\left\{s_{0}(0.5), s_{1}(0.5)\right\}$ \\
$a_{3}$ & $\left\{s_{0}(0.5), s_{1}(0.5)\right\}$ & $\left\{s_{2}(1)\right\}$ & $\left\{s_{0}(0.6), s_{1}(0.4)\right\}$ & $\left\{s_{1}(0.8), s_{2}(0.2)\right\}$ \\
\hline
\end{tabular}

TABle 2: The PLDM $R^{(2)}$ provided by the online media $e_{2}$.

\begin{tabular}{ccccc}
\hline & $c_{1}$ & $c_{2}$ & $c_{3}$ & $c_{4}$ \\
\hline$a_{1}$ & $\left\{s_{-1}(1)\right\}$ & $\left\{s_{2}(1)\right\}$ & $\left\{s_{0}(0.5), s_{1}(0.5)\right\}$ & $\left\{s_{-1}(1)\right\}$ \\
$a_{2}$ & $\left\{s_{2}(0.5), s_{3}(0.5)\right\}$ & $\left\{s_{0}(0.5), s_{1}(0.5)\right\}$ & $\left\{s_{2}(1)\right\}$ & \\
$a_{3}$ & $\left\{s_{0}(1)\right\}$ & $\left\{s_{2}(0.6), s_{3}(0.4)\right\}$ & $\left\{s_{-1}(0.2), s_{0}(0.8)\right\}$ & $\left\{s_{1}(1)\right\}$ \\
\hline
\end{tabular}


Substep 6: using equations (34) and (35), we update experts' weights as follows:

$$
\begin{aligned}
& \lambda^{(1,1)}=0.3406, \\
& \lambda^{(2,1)}=0.3405, \\
& \lambda^{(3,1)}=0.3189 .
\end{aligned}
$$

Let $t:=t+1$ and go to Substep 2 .

After five rounds of consensus iterations (i.e., $t=5$ ), we have the group consensus level $\mathrm{GCD}^{(G, 5)}=0.9505>\eta$. It shows that the adaptive CRP procedure (Algorithm 1 ) ends. So, the terminal iteration step $t=5$. Let $\widetilde{w}^{(j)}=w^{(j, 5)}(j=1,2,3,4), \widetilde{R}^{(G)}=\widehat{R}^{(G, 5)}$, and $\widetilde{\mathrm{GCD}}^{(G)}=$
$\mathrm{GCD}^{(G, 5)}$. Also, let $\tilde{\lambda}^{(k)}=\lambda^{(k, 5)}, \widetilde{R}^{(k)}=\widehat{R}^{(k, 5)}, \widetilde{\mathrm{ICD}}^{(k)}=$ $\operatorname{ICD}^{(k, 5)}, k=1,2,3$.

Then, the final expert weights are $\tilde{\lambda}^{(1)}=0.3655, \tilde{\lambda}^{(2)}=$ 0.3557 , and $\tilde{\lambda}^{(3)}=0.2788$. The final attribute weights are $\widetilde{w}^{(1)}=2106, \widetilde{w}^{(2)}=0.2755, \widetilde{w}^{(3)}=0.2445$, and $\widetilde{w}^{(4)}=$ 0.2694 . The final ICCC degree is $\widetilde{\mathrm{ICD}}^{(1)}=0.0079$, $\widetilde{\mathrm{ICD}}^{(2)}=0.0031$, and $\widetilde{\mathrm{ICD}}^{(3)}=-0.0097$. The final group consensus degree is $\widetilde{\mathrm{GCD}}^{(G)}=0.9505$. The final individual PLDMs $\widetilde{R}^{(k)} \quad(k=1,2,3)$ are $\widetilde{R}^{(1)}=\widehat{R}^{(1)}$, $\widetilde{R}^{(2)}=\widehat{R}^{(2)}$, and

$\widetilde{R}^{(3)}=\left(\begin{array}{cccc}\left\{s_{-0.7321}(0.4767), s_{0.1192}(0.2044), s_{1}(0.3189)\right\} & \left\{s_{1}(0.5), s_{2}(0.5)\right\} & \left\{s_{0}(1)\right\} & \left\{s_{-1}(0.2858), s_{-0.0876}(0.2156), s_{0.8565}(0.4986)\right\} \\ \left\{s_{0}(0.2950), s_{1.8839}(0.5291), s_{3}(0.1759)\right\} & \left\{s_{1}(0.6), s_{2}(0.4)\right\} & \left\{s_{2}(1)\right\} & \left\{s_{-1}(0.8), s_{0}(0.2)\right\} \\ \left\{s_{-1}(0.3333), s_{-0.0876}(0.5000), s_{0.8565}(0.1667)\right\} & \left\{s_{2}(1)\right\} & \left\{s_{-1}(0.3754), s_{-0.0876}(0.4856), s_{0.8565}(0.1390)\right\} & \left\{s_{-2}(1)\right\}\end{array}\right)$.

The individual assessments that are adaptively corrected in sequence include $\widehat{r}_{31}^{(3)}, \widehat{r}_{11}^{(3)}, \widehat{r}_{33}^{(3)}, \widehat{r}_{21}^{(3)}$, and $\widehat{r}_{14}^{(3)}$ in the iterative process.
The final group PLDM $\widetilde{R}^{(G)}$ are
Since the CRP ends, then go to the following selection process.

Step 4: utilizing equations (8) and (9), we obtain the general expected value matrix:

$E\left(\widetilde{R}^{(G)}\right)=\left(E\left(\widetilde{r}_{i j}^{(G)}\right)\right)_{3 \times 4}=\left(\begin{array}{cccc}0.4161 & 0.6950 & 0.5906 & 0.5561 \\ 0.8409 & 0.5811 & 0.8516 & 0.4573 \\ 0.5196 & 0.7969 & 0.4986 & 0.3392\end{array}\right)$

and the general variance value matrix:

$\sigma\left(\widetilde{R}^{(G)}\right)=\left(\sigma\left(\widetilde{r}_{i j}^{(G)}\right)\right)_{3 \times 4}=\left(\begin{array}{llll}0.1066 & 0.0782 & 0.0830 & 0.0660 \\ 0.1243 & 0.0812 & 0.0521 & 0.0517 \\ 0.0908 & 0.0827 & 0.0988 & 0.0754\end{array}\right)$.

Then, using Algorithm 2, we get the weighted general expected value of emergency $a_{i}(i=1,2,3)$ :

$$
E\left(\widetilde{R}_{1}^{(G)}\right)=0.5733, E\left(\widetilde{R}_{2}^{(G)}\right)=0.6686, E\left(\widetilde{R}_{3}^{(G)}\right)=0.5422 \text {, }
$$

and the weighted general variance value of emergency $a_{i}(i=1,2,3)$ :

$$
\sigma\left(\widetilde{R}_{1}^{(G)}\right)=0.0821, \sigma\left(\widetilde{R}_{2}^{(G)}\right)=0.0752, \sigma\left(\widetilde{R}_{3}^{(G)}\right)=0.0864 .
$$

Step 5: according to Algorithm 2, the performances of emergency $a_{i}(i=1,2,3)$ are

$$
\begin{aligned}
& \mathrm{CV}_{1}=0.8748, \\
& \mathrm{CV}_{2}=0.8989, \\
& \mathrm{CV}_{3}=0.8626 .
\end{aligned}
$$

All the emergencies $\left(a_{i}(i=1,2,3)\right)$ are then ranked according to their performance values to get $a_{2}>a_{1}>a_{3}$, where the symbol " $>$ " means "superior to." Therefore, we conclude that the emergency decision-making committee $E=\left\{e_{1}, e_{2}, e_{3}\right\}$, consisting of government departments $\left(e_{1}\right)$, online media $\left(e_{2}\right)$, and netizens $\left(e_{3}\right)$, reaches a consensus that the emergency $a_{2}$ was the most severe Internet public opinion crisis that needs to be prioritized. 
Step 6: end.

6.3. Comparative Analyses. To illustrate the effectiveness and advantages of the proposed approach, two other PLTSsbased MAGDM methods are applied to solving the case in Section 6.1.

6.3.1. Comparison with Aggregation-Based Probabilistic Linguistic MAGDM Method. The first MAGDM method without consideration of CRPs is denoted as Method 2. Specific steps are as follows.

Steps 1 and 2: employ Steps 1-2 of the probabilistic linguistic MAGDM approach with the novel proposed CRP (i.e., Algorithm 2) to obtain the normalized individual PLDMs $\widehat{R}^{(k)}=\left(\widehat{r}_{i j}^{(k)}\right)_{m \times n}(k=1,2, \ldots, h)$.

$$
\widehat{R}^{(G)}=\left(\begin{array}{cccc}
\left\{s_{-1}\left(\frac{7}{15}\right), s_{0}\left(\frac{1}{5}\right), s_{1}\left(\frac{1}{3}\right)\right\} & \left\{s_{1}\left(\frac{13}{30}\right), s_{2}\left(\frac{17}{30}\right)\right\} & \left\{s_{0}\left(\frac{1}{2}\right), s_{1}\left(\frac{1}{2}\right)\right\} & \left\{s_{-1}\left(\frac{1}{3}\right), s_{0}\left(\frac{1}{5}\right), s_{1}\left(\frac{7}{15}\right)\right\} \\
\left\{s_{0}\left(\frac{1}{3}\right), s_{2}\left(\frac{1}{2}\right), s_{3}\left(\frac{1}{6}\right)\right\} & \left\{s_{0}\left(\frac{11}{30}\right), s_{1}\left(\frac{1}{2}\right), s_{2}\left(\frac{2}{15}\right)\right\} & \left\{s_{2}\left(\frac{9}{10}\right), s_{3}\left(\frac{1}{10}\right)\right\} & \left\{s_{-1}\left(\frac{13}{30}\right), s_{0}\left(\frac{17}{30}\right)\right\} \\
\left\{s_{-1}\left(\frac{1}{3}\right), s_{0}\left(\frac{1}{2}\right), s_{1}\left(\frac{1}{6}\right)\right\} & \left\{s_{2}\left(\frac{13}{15}\right), s_{3}\left(\frac{2}{15}\right)\right\} & \left\{s_{-1}\left(\frac{2}{5}\right), s_{0}\left(\frac{7}{15}\right), s_{1}\left(\frac{2}{15}\right)\right\} & \left\{s_{-2}\left(\frac{2}{5}\right), s_{-1}\left(\frac{3}{5}\right)\right\}
\end{array}\right) .
$$

Solving model (M-2), we have the attribute weights: $w^{(1)}=0.2108, \quad w^{(2)}=0.2533, \quad w^{(3)}=0.3206$, and $w^{(4)}=$ 0.2153 . Then, we get the weighted general expected value $\left(E\left(\widehat{R}_{i}^{(G)}\right)\right)$, the weighted general variance value $\left(\sigma\left(\widehat{R}_{i}^{(G)}\right)\right)$, and the performance value $\left(\mathrm{CV}_{i}\right)$ of emergency $a_{i}$ as follows:

$$
\begin{aligned}
E\left(\widehat{R}_{1}^{(G)}\right) & =0.5744, \\
E\left(\widehat{R}_{2}^{(G)}\right) & =0.6773, \\
E\left(\widehat{R}_{3}^{(G)}\right) & =0.5183 ; \\
\sigma\left(\widehat{R}_{1}^{(G)}\right) & =0.0977, \\
\sigma\left(\widehat{R}_{2}^{(G)}\right) & =0.0879, \\
\sigma\left(\widehat{R}_{3}^{(G)}\right) & =0.0975 ; \\
\mathrm{CV}_{1} & =0.8547, \\
\mathrm{CV}_{2} & =0.8852, \\
\mathrm{CV}_{3} & =0.8416 .
\end{aligned}
$$

Since $\mathrm{CV}_{2}>\mathrm{CV}_{1}>\mathrm{CV}_{3}$, then $a_{2}>a_{1}>a_{3}$. Thus, the most severe Internet public opinion crisis that needs to be prioritized is $a_{2}$.

The ranking of Internet public opinion crisis rating obtained by the proposed consensus-driven MAGDM method is consistent with that by Method 2, which verifies the effectiveness of the proposed method in this paper. However, we obtain different performance values of
Step 3: supposing that the weight of each expert is the same, get the collective PLDM $\widehat{R}^{(G)}=\left(\widehat{r}_{i j}^{(G)}\right)_{m \times n}$ by equation (28).

Step 4: solving model (M-2), obtain the attribute weights $w^{(j)}(j=1,2, \ldots, n)$.

Step 5: based on $\widehat{R}^{(G)}$, utilize Algorithm 2 to obtain the weighted general expected value $\left(E\left(\widehat{R}_{i}^{(G)}\right)\right)$ and the weighted general variance value $\left(\sigma\left(\widehat{R}_{i}^{(G)}\right)\right)$ of alternative $a_{i}$, respectively.

Step 6: determine the performance values $\left(\mathrm{CV}_{i}\right)$ of alternative $a_{i}$ by Algorithm 2 and then rank all the alternatives according to the values of $\mathrm{CV}_{i}$ $(i=1,2, \ldots, m)$.

For the case in this paper, using Method 2, we obtain the collective PLDM as follows:

emergencies by different methods. Additionally, we can find that the deviation of performance values between any two emergencies derived by the proposed method is smaller than the one derived by Method 2. The main reason for the difference lies in the fact that the selection process is performed directly in Method 2 without consideration of CRP. Therefore, the solution by the presented MAGDM method is more acceptable for each expert in the group than that by Method 2, illustrating that the presented GDM method is more scientific and democratic by forming the overall satisfaction of the group.

6.3.2. Comparison with the PLTSs-Based MAGDM Method Involving the Only Consensus Improving Technique of Weight-Updating. The second MAGDM method is expressed as Method 3 in which the weight-updating-based CRP (denoted as Algorithm 3) is only integrated into the consensus-driven MAGDM, and the only difference between Algorithms 1 and 3 is no consideration of Step 5 of Algorithm 1 in Algorithm 3. That is, in Algorithm 3, we use the weight-updating technique instead of the integrated consensus improving technique to promote consensus reaching. The main thread of Method 3 is listed below.

Method 3 is applied to solving the case in this paper. To facilitate the comparison of the different methods, it is assumed that all the parameters involved are the same as the decision process in Section 6.2. After 21 rounds of consensus iteration, we obtain $\widehat{\mathrm{GCD}}^{(G)}=\mathrm{GCD}^{(G, 21)}=0.9505>\eta=0.95$, 
TABLE 3: The PLDM $R^{(3)}$ provided by the netizens $e_{3}$.

\begin{tabular}{ccccc}
\hline & $c_{1}$ & $c_{2}$ & $c_{3}$ & $c_{4}$ \\
\hline$a_{1}$ & $\left\{s_{1}(1)\right\}$ & $\left\{s_{1}(0.5), s_{2}(0.5)\right\}$ & $\left\{s_{0}(1)\right\}$ & $\left\{s_{1}(1)\right\}$ \\
$a_{2}$ & $\left\{s_{0}(1)\right\}$ & $\left\{s_{1}(0.6), s_{2}(0.4)\right\}$ & $\left\{s_{2}(1)\right\}$ & $\left\{s_{0}(0.2), s_{1}(0.8)\right\}$ \\
$a_{3}$ & $\left\{s_{-1}(1)\right\}$ & $\left\{s_{2}(1)\right\}$ & $\left\{s_{-1}(1)\right\}$ & $\left\{s_{2}(1)\right\}$ \\
\hline
\end{tabular}

TABLE 4: The individual consensus degree $\operatorname{ICL}_{i}^{(l)}$ of expert $e_{l}$ on emergency $a_{i}(t=0)$.

\begin{tabular}{lccr}
\hline & $e_{1}$ & $e_{2}$ & $e_{3}$ \\
\hline$a_{1}$ & 0.9355 & 0.9326 & 0.9051 \\
$a_{2}$ & 0.9470 & 0.9408 & 0.9132 \\
$a_{3}$ & 0.9262 & 0.9350 & 0.9031 \\
\hline
\end{tabular}

which shows the CRP is terminated. We obtain the final attribute weights: $\widetilde{w}^{(1)}=0.2212, \widetilde{w}^{(2)}=0.2800, \widetilde{w}^{(3)}=0.2666$, and $\widetilde{w}^{(4)}=0.2321$. Also, we get the final expert weights: $\tilde{\lambda}^{(1)}=0.4700, \tilde{\lambda}^{(2)}=0.4437$, and $\tilde{\lambda}^{(3)}=0.0862$. So, the final group PLDM can be obtained as follows:

$$
\widetilde{R}^{(G)}=\left(\begin{array}{cccc}
\left\{s_{-1}(0.6318), s_{0}(0.2820), s_{1}(0.0862)\right\} & \left\{s_{1}(0.4191), s_{2}(0.5809)\right\} & \left\{s_{0}(0.3081), s_{1}(0.6919)\right\} & \left\{s_{-1}(0.0862), s_{0}(0.2820), s_{1}(0.6318)\right\} \\
\left\{s_{0}(0.0862), s_{2}(0.6919), s_{3}(0.2219)\right\} & \left\{s_{0}(0.5039), s_{1}(0.4616), s_{2}(0.0345)\right\} & \left\{s_{2}(0.8590), s_{3}(0.1410)\right\} & \left\{s_{-1}(0.3040), s_{0}(0.6960)\right\} \\
\left\{s_{-1}(0.0862), s_{0}(0.6788), s_{1}(0.2350)\right\} & \left\{s_{2}(0.8225), s_{3}(0.1775)\right\} & \left\{s_{-1}(0.1750), s_{0}(0.6370), s_{1}(0.1880)\right\} & \left\{s_{-2}(0.1802), s_{-1}(0.8198)\right\}
\end{array}\right) .
$$

Then, we get the weighted general expected value $\left(E\left(\widetilde{R}_{i}^{(G)}\right)\right)$, the weighted general variance value $\left(\sigma\left(\widetilde{R}_{i}^{(G)}\right)\right)$, and the performance value $\left(\mathrm{CV}_{i}\right)$ of emergency $a_{i}$ as follows:

$$
\begin{aligned}
E\left(\widetilde{R}_{1}^{(G)}\right) & =0.5791 \\
E\left(\widetilde{R}_{2}^{(G)}\right) & =0.6794 \\
E\left(\widetilde{R}_{3}^{(G)}\right) & =0.5605 \\
\sigma\left(\widetilde{R}_{1}^{(G)}\right) & =0.0821 \\
\sigma\left(\widetilde{R}_{2}^{(G)}\right) & =0.0725 \\
\sigma\left(\widetilde{R}_{3}^{(G)}\right) & =0.0864 \\
\mathrm{CV}_{1} & =0.8759 \\
\mathrm{CV}_{2} & =0.9036 \\
\mathrm{CV}_{3} & =0.8665 .
\end{aligned}
$$

Since $\mathrm{CV}_{2}>\mathrm{CV}_{1}>\mathrm{CV}_{3}$, then the consensus ranking is $a_{2}>a_{1}>a_{3}$, which implies that $a_{2}$ is the most severe Internet public opinion crisis. The consensus ranking is consistent with the one derived by Algorithm 2 while there is a slight difference in the performance values of each emergency obtained by two different consensus improving procedures (i.e., Algorithms 1 and 3). The group consensus level with the adaptive updating of expert weights by the above different consensus improving strategies is shown in Figures 4 and 5, respectively. By comparing Figures 4 with 5, the common feature of the two consensus improving processes can be concluded that the same acceptable group consensus degree (i.e., a consensus threshold $\eta=0.95$ established a priori) is achieved by punishing the weight of the expert $e_{3}$ with less ICCC and rewarding the weight of the experts $e_{1}$ and $e_{2}$. However, the number of consensus iterations using Algorithm 3 is extremely larger than the one using Algorithm 1, which shows that the consensus improving algorithm proposed in this paper is better in terms of consensus efficiency. The main reason is that the adaptive assessment-adjusting model in Section 5.3 is a useful supplement to the rapid achievement of group consensus. 
TABLE 5: The consensus degree $\mathrm{CL}_{i}$ of all experts on emergency $a_{i}(t=0)$.

\begin{tabular}{lccc}
\hline$a_{i}$ & $a_{1}$ & $a_{2}$ & $a_{3}$ \\
\hline $\mathrm{CL}_{i}$ & 0.9244 & 0.9337 & 0.9214 \\
\hline
\end{tabular}

TABLE 6: The consensus degree $\mathrm{CL}_{i}^{(l)}$ on emergency $a_{i}$ without expert $e_{l}(t=0)$.

\begin{tabular}{lccr}
\hline & $e_{1}$ & $e_{2}$ & $e_{3}$ \\
\hline$a_{1}$ & 0.9188 & 0.9203 & 0.9340 \\
$a_{2}$ & 0.9270 & 0.9301 & 0.9439 \\
$a_{3}$ & 0.9191 & 0.9146 & 0.9306 \\
\hline
\end{tabular}

Steps 1 and 2: the same as Algorithm 2 (i.e., the probabilistic linguistic MAGDM approach with the novel CRP).

Step 3: use Algorithm 3 to implement the consensus process. If the CRP ends, then go to Step 4.

Steps 4-6: the same as Algorithm 2.

Algorithm 3: The weight-updating-based CRP.

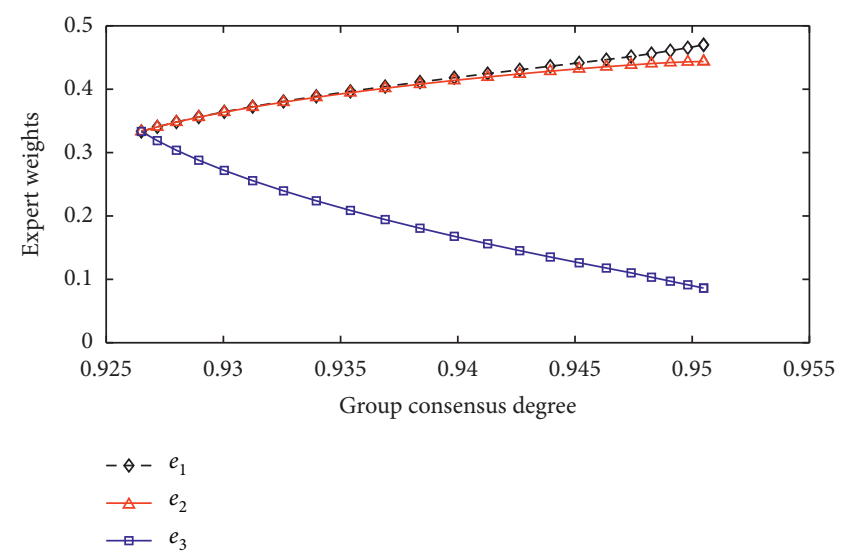

Figure 4: Group-consensus levels with the updating of weights of experts by Algorithm 3.

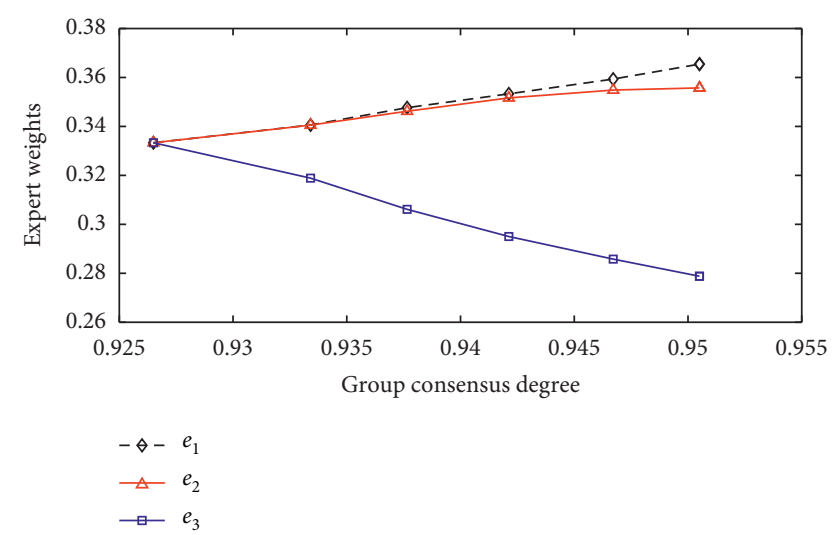

Figure 5: Group-consensus levels with the updating of weights of experts by Algorithm 1.

\section{Concluding Remarks}

To build consensus in probabilistic linguistic MAGDM with PLDMs, this paper proposed an adaptive consensus reaching model with an integrated improving strategy based on PLWasserstein distance. Compared to the existing consensus reaching models with PLDMs, the proposed model has the following advantages. (a) By applying the PL-Wasserstein distance, the group consensus degree and the ICCC degree, two key indicators of conducting the adaptive CRP, can be accurately measured. (b) The presented CRP is an adaptive CRP that can not only reduce the reliance on the moderator but also eliminate the subjective influence of the moderator in CRP. (c) The integrated consensus improving strategy which considers both weight-updating (i.e., dynamic weights of experts and attributes) and assessment-adjusting is employed to greatly improve the consensus efficiency of MAGDM. Especially for emergency MAGDM problems, this treatment can be very beneficial for rapid emergency response.

Meanwhile, we find the following limitations of the paper, which need to be overcome in future research. (a) Different people have different understandings regarding the same word, which is characterized by personalized individual semantics (PIS) [68, 69]. However, we failed to consider PIS of each expert in constructing the consensus model for MAGDM with probabilistic linguistic assessment information. (b) Moreover, the psychological behaviors (such as regret and disappointment [70], bounded confidence [71], and overconfidence [72]) of emergency experts are often implicit in the crisis evaluation of the online public opinion. However, we did not integrate the behavior of decision makers into the construction of the consensus model. Thus, for evaluating the network public opinion 
emergencies, we argue that there are two interesting research paths for the future. (a) Consensus issues should be investigated in the PIS-based emergency MAGDM framework. (b) Decision makers' behaviors ought to be considered when designing consensus-driven emergency MAGDM approaches.

\section{Data Availability}

The data used to support the findings of this study are included within this article. They are also available from the corresponding author upon request.

\section{Conflicts of Interest}

The authors declare that there are no conflicts of interest regarding the publication of this paper.

\section{Acknowledgments}

This study was supported by the Humanities and Social Sciences Foundation of Ministry of Education of China (nos. 18YJC630249 and 16YJC630077), the National Natural Science Foundation of China (nos. 72074001, 71601002, and 71704001), the China Postdoctoral Science Foundation (no. 2019M660411), the Anhui Provincial Natural Science Foundation (no. 1708085MG168), and the Provincial Humanities and Social Science Research Project of Anhui Colleges (no. SK2018A0605).

\section{References}

[1] S. Fang, N. Zhao, N. Chen, F. Xiong, and Y. Yi, "Analyzing and predicting network public opinion evolution based on group persuasion force of populism," Physica A: Statistical Mechanics and Its Applications, vol. 525, pp. 809-824, 2019.

[2] X. Ma, W. Liu, X. Zhou et al., "Evolution of online public opinion during meteorological disasters," Environmental Hazards, vol. 19, no. 4, pp. 375-397, 2020.

[3] L. Zhang, C. Su, Y. Jin, M. Goh, and Z. Wu, "Cross-network dissemination model of public opinion in coupled networks," Information Sciences, vol. 451-452, pp. 240-252, 2018.

[4] Z. Y. Wang, X. D. Liu, and S. T. Zhang, "A new decision method for public opinion crisis with the intervention of risk perception of the public," Complexity, vol. 2019, Article ID 9527218, 14 pages, 2019.

[5] X. Xu, X. Yin, and X. Chen, "A large-group emergency risk decision method based on data mining of public attribute preferences," Knowledge-Based Systems, vol. 163, pp. 495-509, 2019.

[6] P. Li and C. P. Wei, "An emergency decision-making method based on DS evidence theory for probabilistic linguistic term sets," International Journal of Disaster Risk Reduction, vol. 37, Article ID 101178, 2019.

[7] X. F. Ding and H. C. Liu, "A new approach for emergency decision-making based on zero-sum game with Pythagorean fuzzy uncertain linguistic variables," International Journal of Intelligent Systems, vol. 34, no. 7, pp. 1667-1684, 2019.

[8] S. Zhang, J. Zhu, X. Liu, Y. Chen, and Z. Ma, "Adaptive consensus model with multiplicative linguistic preferences based on fuzzy information granulation," Applied Soft Computing, vol. 60, pp. 30-47, 2017.
[9] X. Xu, Q. Zhang, and X. Chen, "Consensus-based non-cooperative behaviors management in large-group emergency decision-making considering experts' trust relations and preference risks," Knowledge-Based Systems, vol. 190, Article ID 105108, 2020.

[10] H. Wang, Z. Xu, and X.-J. Zeng, "Modeling complex linguistic expressions in qualitative decision making: an overview," Knowledge-Based Systems, vol. 144, pp. 174-187, 2018.

[11] R. M. Rodriguez, L. Martínez, and F. Herrera, "Hesitant fuzzy linguistic term sets for decision making," IEEE Transactions on Fuzzy Systems, vol. 20, no. 1, pp. 109-119, 2011.

[12] H. Wang, "Extended hesitant fuzzy linguistic term sets and their aggregation in group decision making," International Journal of Computational Intelligence Systems, vol. 8, no. 1, pp. 14-33, 2015.

[13] J. H. Wang and J. Y. Hao, "A new version of 2-tuple fuzzy linguistic representation model for computing with words," IEEE Transactions on Fuzzy Systems, vol. 14, no. 3, pp. 435445, 2006.

[14] G. Zhang, Y. Dong, and Y. Xu, "Consistency and consensus measures for linguistic preference relations based on distribution assessments," Information Fusion, vol. 17, pp. 46-55, 2014.

[15] Z. B. Wu and J. P. Xu, "Possibility distribution-based approach for MAGDM with hesitant fuzzy linguistic information," IEEE Transactions on Cybernetics, vol. 46, no. 3, pp. 694-705, 2015.

[16] Z.-S. Chen, K.-S. Chin, N.-Y. Mu, S.-H. Xiong, J.-P. Chang, and Y. Yang, "Generating HFLTS possibility distribution with an embedded assessing attitude," Information Sciences, vol. 394-395, pp. 141-166, 2017.

[17] Z.-S. Chen, K.-S. Chin, L. Martinez, and K.-L. Tsui, "Customizing semantics for individuals with attitudinal HFLTS possibility distributions," IEEE Transactions on Fuzzy Systems, vol. 26, no. 6, pp. 3452-3466, 2018.

[18] Z.-S. Chen, Y. Yang, X.-J. Wang, K.-S. Chin, and K.-L. Tsui, "Fostering linguistic decision-making under uncertainty: a proportional interval type-2 hesitant fuzzy TOPSIS approach based on Hamacher aggregation operators and andness optimization models," Information Sciences, vol. 500, pp. 229258, 2019.

[19] Z.-S. Chen, M. Li, W.-T. Kong, and K.-S. Chin, "Evaluation and selection of HazMat transportation alternatives: a PHFLTS- and TOPSIS-integrated multi-perspective approach," International Journal of Environmental Research and Public Health, vol. 16, no. 21, p. 4116, 2019.

[20] Z.-S. Chen, K.-S. Chin, Y.-L. Li, and Y. Yang, "Proportional hesitant fuzzy linguistic term set for multiple criteria group decision making," Information Sciences, vol. 357, pp. 61-87, 2016.

[21] Q. Pang, H. Wang, and Z. Xu, "Probabilistic linguistic term sets in multi-attribute group decision making," Information Sciences, vol. 369, pp. 128-143, 2016.

[22] Y. Wu, Z. Zhang, G. Kou et al., "Distributed linguistic representations in decision making: taxonomy, key elements and applications, and challenges in data science and explainable artificial intelligence," Information Fusion, vol. 65, pp. 165$178,2021$.

[23] H. Liao, X. Mi, and Z. Xu, "A survey of decision-making methods with probabilistic linguistic information: bibliometrics, preliminaries, methodologies, applications and future directions," Fuzzy Optimization and Decision Making, vol. 19, no. 1, pp. 81-134, 2020. 
[24] J. Kacprzyk and M. Fedrizzi, "A "soft” measure of consensus in the setting of partial (fuzzy) preferences," European Journal of Operational Research, vol. 34, no. 3, pp. 316-325, 1988.

[25] F. Herrera, E. Herrera-Viedma, and J. L. Verdegay, "A model of consensus in group decision making under linguistic assessments," Fuzzy Sets and Systems, vol. 78, no. 1, pp. 73-87, 1996.

[26] Z. Wu, B. Jin, and J. Xu, "Local feedback strategy for consensus building with probability-hesitant fuzzy preference relations," Applied Soft Computing, vol. 67, pp. 691-705, 2018.

[27] J. Wu, Q. Sun, H. Fujita, and F. Chiclana, "An attitudinal consensus degree to control the feedback mechanism in group decision making with different adjustment cost," KnowledgeBased Systems, vol. 164, pp. 265-273, 2019.

[28] I. Palomares, F. J. Estrella, L. Martínez, and F. Herrera, "Consensus under a fuzzy context: taxonomy, analysis framework AFRYCA and experimental case of study," Information Fusion, vol. 20, pp. 252-271, 2014.

[29] B. Zhang, Y. Dong, and E. Herrera-Viedma, "Group decision making with heterogeneous preference structures: an automatic mechanism to support consensus reaching," Group Decision and Negotiation, vol. 28, no. 3, pp. 585-617, 2019.

[30] Z. Gong, W. Guo, E. Herrera-Viedma, Z. Gong, and G. Wei, "Consistency and consensus modeling of linear uncertain preference relations," European Journal of Operational Research, vol. 283, no. 1, pp. 290-307, 2020.

[31] Á. Labella, Y. Liu, R. M. Rodríguez, and L. Martínez, “Analyzing the performance of classical consensus models in large scale group decision making: a comparative study," Applied Soft Computing, vol. 67, pp. 677-690, 2018.

[32] Y. Dong, Q. Zha, H. Zhang et al., "Consensus reaching in social network group decision making: research paradigms and challenges," Knowledge-Based Systems, vol. 162, pp. 3-13, 2018.

[33] I. J. Pérez, F. J. Cabrerizo, S. Alonso, Y. C. Dong, F. Chiclana, and E. Herrera-Viedma, "On dynamic consensus processes in group decision making problems," Information Sciences, vol. 459 , pp. 20-35, 2018.

[34] H. Zhang, S. Zhao, G. Kou, C.-C. Li, Y. Dong, and F. Herrera, "An overview on feedback mechanisms with minimum adjustment or cost in consensus reaching in group decision making: research paradigms and challenges," Information Fusion, vol. 60, pp. 65-79, 2020.

[35] X. Wu and H. Liao, "A consensus-based probabilistic linguistic gained and lost dominance score method," European Journal of Operational Research, vol. 272, no. 3, pp. 1017-1027, 2019.

[36] M. J. del Moral, F. Chiclana, J. M. Tapia, and E. HerreraViedma, "A comparative study on consensus measures in group decision making," International Journal of Intelligent Systems, vol. 33, no. 8, pp. 1624-1638, 2018.

[37] X.-B. Mao, M. Wu, J.-Y. Dong, S.-P. Wan, and Z. Jin, “A new method for probabilistic linguistic multi-attribute group decision making: application to the selection of financial technologies," Applied Soft Computing, vol. 77, pp. 155-175, 2019.

[38] X. K. Wang, J. Q. Wang, and H. Y. Zhang, "Distance-based multicriteria group decision-making approach with probabilistic linguistic term sets," Expert Systems, vol. 36, no. 2, Article ID e12352, 2019.

[39] Y. Zhang, Z. Xu, H. Wang, and H. Liao, "Consistency-based risk assessment with probabilistic linguistic preference relation," Applied Soft Computing, vol. 49, pp. 817-833, 2016.
[40] X. Wu, H. Liao, Z. Xu, A. Hafezalkotob, and F. Herrera, "Probabilistic linguistic MULTIMOORA: a multicriteria decision making method based on the probabilistic linguistic expectation function and the improved Borda rule," IEEE Transactions on Fuzzy Systems, vol. 26, no. 6, pp. 3688-3702, 2018.

[41] $\mathrm{Z}$. Wu and J. Xu, "A consensus model for large-scale group decision making with hesitant fuzzy information and changeable clusters," Information Fusion, vol. 41, pp. 217-231, 2018.

[42] X.-h. Xu, Z.-j. Du, and X.-h. Chen, "Consensus model for multi-criteria large-group emergency decision making considering non-cooperative behaviors and minority opinions," Decision Support Systems, vol. 79, pp. 150-160, 2015.

[43] M. Tang, H. Liao, J. Xu, D. Streimikiene, and X. Zheng, "Adaptive consensus reaching process with hybrid strategies for large-scale group decision making," European Journal of Operational Research, vol. 282, no. 3, pp. 957-971, 2020.

[44] M. Gupta, "Consensus building process in group decision making-an adaptive procedure based on group dynamics," IEEE Transactions on Fuzzy Systems, vol. 26, no. 4, pp. 1923-1933, 2018.

[45] S.-M. Chen and B.-H. Tsai, “Autocratic decision making using group recommendations based on intervals of linguistic terms and likelihood-based comparison relations," IEEE Transactions on Systems, Man, and Cybernetics: Systems, vol. 45, no. 2, pp. 250-259, 2015.

[46] Y. Xu, X. Liu, and L. Xu, "A dynamic expert contributionbased consensus model for hesitant fuzzy group decision making with an application to water resources allocation selection," Soft Computing, vol. 24, no. 6, pp. 4693-4708, 2020.

[47] Y. Dong, H. Zhang, and E. Herrera-Viedma, "Integrating experts' weights generated dynamically into the consensus reaching process and its applications in managing non-cooperative behaviors," Decision Support Systems, vol. 84, pp. 1-15, 2016.

[48] Z. Shi, X. Wang, I. Palomares, S. Guo, and R.-X. Ding, "A novel consensus model for multi-attribute large-scale group decision making based on comprehensive behavior classification and adaptive weight updating," Knowledge-Based Systems, vol. 158, pp. 196-208, 2018.

[49] X. Liu, Y. Xu, R. Montes, and F. Herrera, "Social network group decision making: managing self-confidence-based consensus model with the dynamic importance degree of experts and trust-based feedback mechanism," Information Sciences, vol. 505, pp. 215-232, 2019.

[50] X. Wu and H. Liao, "An approach to quality function deployment based on probabilistic linguistic term sets and ORESTE method for multi-expert multi-criteria decision making," Information Fusion, vol. 43, pp. 13-26, 2018.

[51] H. Zhang, Y. Dong, F. Chiclana, and S. Yu, "Consensus efficiency in group decision making: a comprehensive comparative study and its optimal design," European Journal of Operational Research, vol. 275, no. 2, pp. 580-598, 2019.

[52] Z. Xu, "Deviation measures of linguistic preference relations in group decision making," Omega, vol. 33, no. 3, pp. 249-254, 2005.

[53] X. Gou, Z. Xu, and H. Liao, "Multiple criteria decision making based on Bonferroni means with hesitant fuzzy linguistic information," Soft Computing, vol. 21, no. 21, pp. 6515-6529, 2017.

[54] S. Bobkov and M. Ledoux, "One-dimensional empirical measures, order statistics, and kantorovich transport distances," Memoirs of the American Mathematical Society, vol. 261, no. 1259, pp. 1-126, 2019. 
[55] R. L. Dobrushin, "Prescribing a system of random variables by conditional distributions," Theory of Probability \& Its Applications, vol. 15, no. 3, pp. 458-486, 1970.

[56] C. Villani, Optimal Transport: Old and New, Springer-Verlag, Berlin, Germany, 2009.

[57] V. M. Panaretos and Y. Zemel, "Statistical aspects of Wasserstein distances," Annual Review of Statistics and Its Application, vol. 6, no. 1, pp. 405-431, 2019.

[58] A. L. Gibbs and F. E. Su, "On choosing and bounding probability metrics," International Statistical Review/Revue Internationale de Statistique, vol. 70, no. 3, pp. 419-435, 2002.

[59] L. Kantorovich and G. Rubinstein, On a Space of Completely Additive Functions (Russian), Vol. 13, Leningrad University, St. Petersburg, Russia, 1958.

[60] Y. Rubner, C. Tomasi, and L. J. Guibas, "The earth mover's distance as a metric for image retrieval," International Journal of Computer Vision, vol. 40, no. 2, pp. 99-121, 2000.

[61] X. Wan, "A novel document similarity measure based on earth mover's distance," Information Sciences, vol. 177, no. 18, pp. 3718-3730, 2007.

[62] M. Arjovsky, S. Chintala, and L. Bottou, "Wasserstein GAN," 2017, https://arxiv.org/abs/1701.07875.

[63] Y. M. Wang and Y. Luo, "Integration of correlations with standard deviations for determining attribute weights in multiple attribute decision making," Mathematical and Computer Modelling, vol. 51, no. 1-2, pp. 1-12, 2010.

[64] X. Gou and Z. Xu, "Novel basic operational laws for linguistic terms, hesitant fuzzy linguistic term sets and probabilistic linguistic term sets," Information Sciences, vol. 372, pp. 407427, 2016.

[65] M. Tang, X. Zhou, H. Liao, J. Xu, H. Fujita, and F. Herrera, "Ordinal consensus measure with objective threshold for heterogeneous large-scale group decision making," Knowledge-Based Systems, vol. 180, pp. 62-74, 2019.

[66] Y. Xu, W. Zhang, and H. Wang, "A conflict-eliminating approach for emergency group decision of unconventional incidents," Knowledge-Based Systems, vol. 83, pp. 92-104, 2015.

[67] X. Liu, Z. Wang, and A. Hetzler, "HFMADM method based on nondimensionalization and its application in the evaluation of inclusive growth," Journal of Business Economics and Management, vol. 18, no. 4, pp. 726-744, 2017.

[68] C.-C. Li, Y. Dong, F. Herrera, E. Herrera-Viedma, and L. Martínez, "Personalized individual semantics in computing with words for supporting linguistic group decision making. An application on consensus reaching," Information Fusion, vol. 33, pp. 29-40, 2017.

[69] H. Zhang, Y. Dong, J. Xiao, F. Chiclana, and E. HerreraViedma, "Personalized individual semantics-based approach for linguistic failure modes and effects analysis with incomplete preference information," IISE Transactions, vol. 52, no. 11, pp. 1275-1296, 2020.

[70] S. Zhang, J. Zhu, X. Liu, and Y. Chen, "Regret theory-based group decision-making with multidimensional preference and incomplete weight information," Information Fusion, vol. 31, pp. 1-13, 2016.

[71] H. Zhang, J. Xiao, and Y. Dong, "Integrating a consensusreaching mechanism with bounded confidences into failure mode and effect analysis under incomplete context," Knowledge-Based Systems, vol. 183, Article ID 104873, 2019.

[72] X. Liu, Y. Xu, and F. Herrera, "Consensus model for largescale group decision making based on fuzzy preference relation with self-confidence: detecting and managing overconfidence behaviors," Information Fusion, vol. 52, pp. 245-256, 2019. 